\title{
Notoginsenoside R1 for Organs Ischemia/Reperfusion Injury: A Preclinical Systematic Review
}

\author{
Qiang Tong ${ }^{1+}$, Peng-chong Zhu ${ }^{1+}$, Zhuang Zhuang ${ }^{1}$, Li-hui Deng ${ }^{1}$, Zi-hao Wang ${ }^{1}$, \\ Hua Zeng ${ }^{2}$, Guo-qing Zheng ${ }^{2 *}$ and Yan Wang ${ }^{1 *}$ \\ ${ }^{1}$ Department of Cardiology, The Second Affiliated Hospital and Yuying Children's Hospital of Wenzhou Medical University, \\ Wenzhou, China, ${ }^{2}$ Department of Neurology, The Second Affiliated Hospital and Yuying Children's Hospital of Wenzhou \\ Medical University, Wenzhou, China
}

Notoginsenoside R1 (NGR1) exerts pharmacological actions for a variety of diseases such as myocardial infarction, ischemic stroke, acute renal injury, and intestinal injury. Here, we

OPEN ACCESS

Edited by:

Yue Liu,

Xiyuan Hospital, China

Reviewed by:

Yun K. Tam,

Sinoveda Canada Inc.,

Canada

Sefirin Djiogue,

University of Yaounde I,

Cameroon

*Correspondence:

Guo-qing Zheng gq_zheng@sohu.com

Yan Wang

wywzchina@sina.com

tThese authors have contributed equally to this work

Specialty section: This article was submitted to Ethnopharmacology,

a section of the journal Frontiers in Pharmacology

Received: 13 June 2019 Accepted: 17 September 2019 Published: 17 October 2019

Citation:

Tong Q, Zhu P-c, Zhuang Z, Deng L-h, Wang Z-h, Zeng $H$, Zheng G-q and Wang Y (2019) Notoginsenoside $R 1$ for Organs Ischemia/Reperfusion Injury: A Preclinical Systematic Review.

Front. Pharmacol. 10:1204. doi: 10.3389/fphar.2019.01204 conducted a preclinical systematic review of NGR1 for ischemia reperfusion (I/R) injury. Eight databases were searched from their inception to February 23rd, 2019; Review Manager 5.3 was applied for data analysis. CAMARADES 10-item checklist and cell 10-item checklist were used to evaluate the methodological quality. Twenty-five studies with 304 animals and 124 cells were selected. Scores of the risk of bias in animal studies ranged from 3 to 8 , and the cell studies ranged from 3 to 5 . NGR1 had significant effects on decreasing myocardial infarct size in myocardial I/R injury, decreasing cerebral infarction volume and neurologic deficit score in cerebral I/R injury, decreasing serum creatinine in renal I/R injury, and decreasing Park/Chiu score in intestinal I/R injury compared with controls (all $P<0.05$ or $P<0.01$ ). The multiple organ protection of NGR1 after I/R injury is mainly through the mechanisms of antioxidant, anti-apoptosis, and anti-inflammatory, promoting angiogenesis and improving energy metabolism. The findings showed the organ protection effect of NGR1 after I/R injury, and NGR1 can potentially become a novel drug candidate for ischemic diseases. Further translation studies are needed.

Keywords: notoginsenoside R1, ischemia, reperfusion, organ, preclinical systematic review, meta-analysis

\section{INTRODUCTION}

Ischemia and reperfusion (I/R) injury, featuring as an interruption of organ blood flow and the following re-oxygenation after reperfusion, is a common pathological phenomenon in ischemic diseases. These mainly include myocardial infarction (MI), ischemic stroke, acute renal injury, and intestinal injury (Eltzschig and Eckle 2011). Although these pathological processes are involved in a variety of diseases, these diseases share common molecular mechanisms. The main mechanisms of I/R injury include inflammation (Chen and Nuñez, 2010), oxidative stress (Ohsawa et al., 2007), apoptosis (Shiva et al., 2007), energy metabolism disorder (Wang and Ma, 2018), microvascular dysfunction (Eltzschig and Collard, 2004), and leucocyte-endothelial cell adhesion (Eltzschig and Eckle 2011). Owing to the chronic organ injury, the ischemia organ will ultimately develop into the pathological outcomes with tissue fibrosis and organ failure (Friedman et al., 2013). In 2016, the two main ischemic diseases, ischemic heart disease (IHD) and stroke, were the leading causes of human death globally, accounting for more than $85.1 \%$ of all cardiovascular and cerebrovascular 
diseases and death (Murray Christopher 2017). Over the past three decades, great progress has been made in the therapy of ischemic diseases, especially in MI and ischemic stroke. Nevertheless, there exist disadvantages in safety and efficacy in main and promising therapy approaches. In acute management, the timely revascularization therapies such as percutaneous coronary intervention (PCI) and thrombolysis can recover the supply of oxygen and blood for ischemic organs or tissues. However, irreversible ischemia from delayed administration and reperfusion injury can result in chronic organ failure and a shortened lifespan (Alex et al., 2017). Currently antiplatelet drugs have good effects on anti-platelet adhesion and aggregation in clinic, but they have little efficacy in energy metabolism disorder and oxidative stress (Han et al., 2017). Studies have shown that ischemic preconditioning and ischemic postconditioning can reduce MI size caused by I/R injury, whereas the mechanism of the conditioning phenomenon with the most robust cardioprotective procedure through interventions reducing MI size is still largely unknown in the human heart (Heusch and Rassaf 2016; Heusch and Gersh, 2016; Heusch, 2017). Currently, recanalization therapy for acute ischemic stroke (AIS) is mainly through recombinant tissue plasminogen activator (rt-PA). However, rt-PA is accepted by a minority of patients due to narrow time window of thrombolysis and hemorrhage (Hacke et al., 2004; Khandelwal et al., 2016). Unfortunately, the reocclusion after thrombolysis led to neurological function impairment and higher in-hospital mortality (Lee et al., 2001). Considering the limitations of these therapies, we need to seek new therapy to improve organ damage induced by I/R injury.

Panax notoginseng ( $P$. notoginseng), one of the most valuable traditional Chinese medicine (TCM), is derived from the roots and rhizomes of P. notoginseng (Burkill) F.H. Chen (Ng, 2006; Peng et al., 2018). Over the past several centuries, $P$. notoginseng showed good efficacy in controlling internal and external bleeding and improving blood stasis (Wang T. et al., 2016). With the advancing of pharmacology, the studies of $P$. notoginseng demonstrate that it is widely used in cardiovascular diseases (CVDs) mainly because of its vasodilatory and antihypertensive functions (Yang et al., 2014). Notoginsenoside R1 (NGR1) (the specific chemical structure of NGR1 is shown in Figure 1) is the main effective component isolated from $P$. notoginseng, and NGR1 belongs to protopanaxatriol (PPT) type of saponins (Leung et al., 2007). Previous studies indicated that NGR1 is easily dissolved in water, but shows low bio-availability and poor permeability in the gastrointestinal tract (Liang and Hua, 2005; Liu et al., 2009; Ruan et al., 2010). In absorption property, Liang et al. (2005) revealed that the optimal site for absorption of NGR1 is the upper extremity of the intestine. Guo et al. (2014) first described the detection of NGR1 in brain tissue by the way of nasally applying drugs. To improve the oral bioavailability of NGR1, sodium N-[8-(2-hydroxybenzoyl) amino] caprylate (SNAC, a novel absorption enhancer) was used (Li et al., 2018). It has been reported that NGR1 significantly improves prognosis of other disease models such as atherosclerosis, diabetic nephropathy, and diabetic cardiomyopathy (Su et al., 2015; Zhang et al., 2018; Zhang B. et al., 2019). NGR1 also plays a protective role in ischemic diseases (Liu et al., 2010; Li et al.,

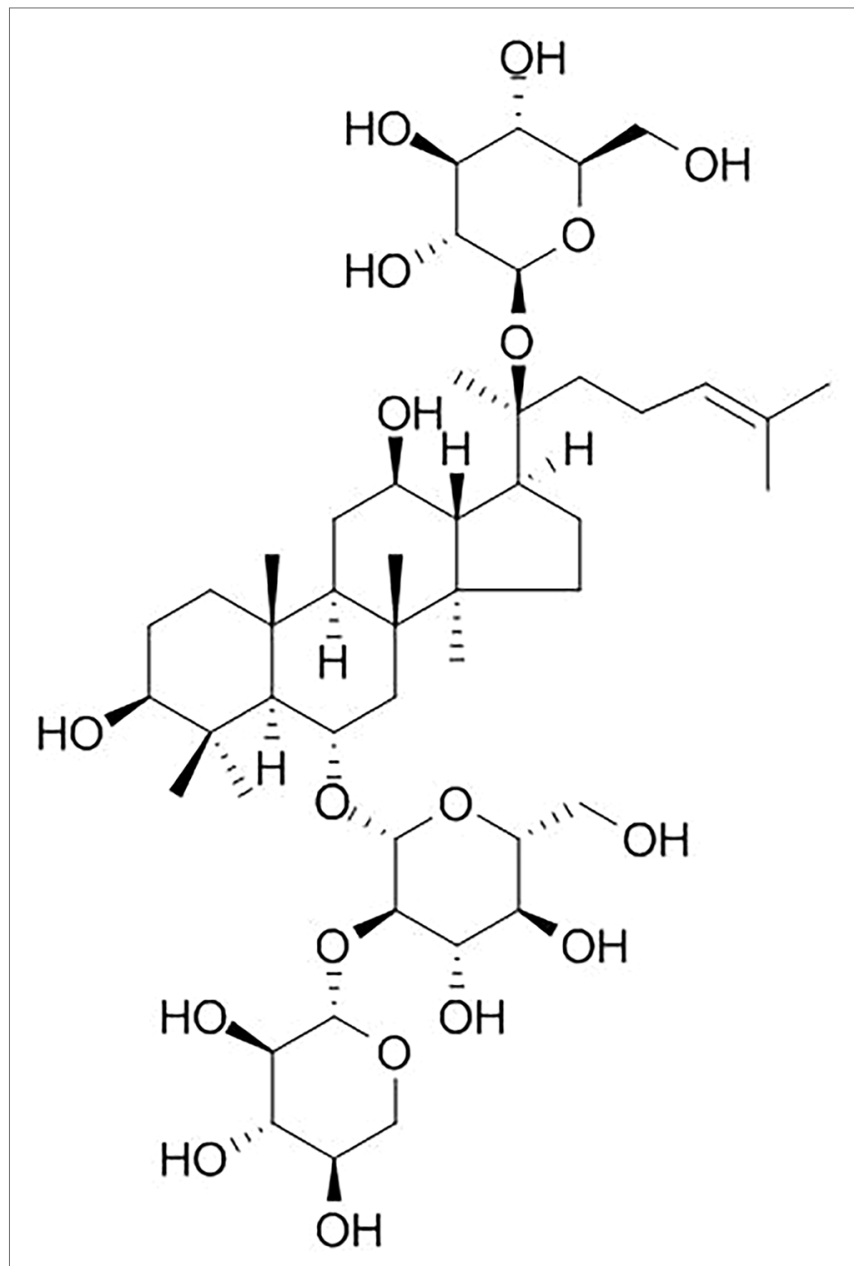

FIGURE 1 | Chemical structure of notoginsenoside R1.

2014; Yu et al., 2016; Tu et al., 2018). Although NGR1 has been widely used for the treatment of ischemic diseases, the efficacy and mechanisms of NGR1 for ischemic organs such as heart, cerebral, kidney, intestinal, and liver have not been systematically analyzed. Animal experiment, the most important approach of basic research, is a bridge between the bench and bedside (Olesen et al., 2012). The conclusions derived from preclinical studies are of little evaluation, and using these conclusions as inadequate evidence for conducting clinical trials has resulted in a high cost in clinical research or withdrawal of the drug from the market later (Perel et al., 2006). Systematic reviews (SRs) are usually used in clinical study, which provided available resources for performing clinical practice guidelines and policies. SR of preclinical studies also plays a significant role in many aspects, including: 1) improving the methodological quality of studies, 2) choosing suitable animal models, 3 ) translating the experimental data from preclinical to clinical, and 4) implementing the 3Rs (reduction, replacement, and refinement) (De Vries et al., 2014). In addition, SR of preclinical experiments is of significance to elucidate the mechanism and treatment of human diseases (Sena et al., 2014). SR can further evaluate the preclinical evidence objectively and reduce the bias of experimental results (Roberts 
et al., 2002; Perel et al., 2006). SRs for preclinical study that could offer crucial information for clinical research are quite scarce (Judith et al., 2014; Tsujimoto et al., 2017). Thus, we conducted an SR on preclinical studies of NGR1 for I/R injury.

\section{METHODS}

\section{Search Strategy}

We searched PubMed, Cochrane Library, EMBASE, Web of Science, Chinese National Knowledge Infrastructure (CNKI), Wanfang Data Information Site, VIP information database, and Chinese Biomedical Literature Database from their inception to February 23rd, 2019. The following terms were used: 1) "notoginsenoside" AND "infarction OR ischem OR reperfusion" limited on animals; and 2) "notoginsenoside" AND "Oxygenglucose deprivation OR hypoxia” limited on cells.

\section{Inclusion Criteria/Exclusion Criteria}

Inclusion criteria were prespecified as follows: 1) experimental animal models of $\mathrm{I} / \mathrm{R} ; 2$ ) experimental cell models established by oxygen and glucose deprivation/reoxygenation (OGD/R); 3) treatment group received the NGR1 therapy merely; 4) control group received vehicle, non-functional liquid with equal volume, no treatment or positive control; 5) the primary outcome measures were $\mathrm{MI}$ size, creatine kinase $(\mathrm{CK})$ or creatine kinase isoenzymes $\mathrm{MB}$ (CKMB), left ventricular ejection fraction (LVEF) and cardiac troponin I/T (cTnI/T) in myocardial I/R studies; cerebral infarct volume and neurologic deficit score in cerebral $I / R$ studies; myeloperoxidase (MPO), glucose/water clearance and Park/Chiu score in intestinal I/R studies; MPO, serum creatinine (Scr), glomerular filtration rate (GFR), and blood urea nitrogen (BUN) in renal I/R studies, and alanine aminotransferase (ALT) and aspartate aminotransferase (AST) in liver I/R studies; cell viability, lactate dehydrogenase $(\mathrm{LDH})$, superoxide dismutase (SOD), malondialdehyde (MDA), apoptosis rate and/or TUNEL positive rate in cell models; 6 ) the secondary outcome measures were mechanisms of NGR1 intervention in both animal and cell models. Exclusion criteria were prespecified as follows: 1) treatment group received non-NGR1;2) comparing NGR1 with other herbal medicine or herbal active compounds; 3) no control group; 4) master dissertation or doctoral dissertation; 5) case report or review; 6) NGR1 for other disease models; 7) NGR1 in combination with other drugs; 8) duplicate publication.

\section{Data Extraction}

A data extraction form was used to collect the following items from each included study: 1) first author, year of publication; 2) detailed information about the experimental subjects such as animal species, number, sex and weight, and cell number, organism, age, tissue, and primary/subcultured; 3) administration method and duration of NGR1 treatment; 4) the types and administration methods of anesthetics; 5) the outcome measures including type, timing, and mean and standard deviations.

Only the last time point and the highest dose were recorded if there were many different time points of outcome measures or the experimental animals received different doses of the drug. The data were measured by the digital ruler software if the data were presented with graphs. Further information was retrieved by contacting with the authors when the primary data were incomplete.

\section{Assessment of the Risk of Bias}

Minor modified CAMARADES 10-item scale was used to assess the risk of bias in animal studies (Macleod et al., 2004). The modified item is the use of anesthetic with no intrinsic organprotective activity. Our newly developed scale specially designed for cell studies was used for the assessment of risk of bias in cell experiments (Bao et al., 2018).

\section{Statistical Analysis}

All data analysis was implemented by RevMan 5.3 (https:// community.cochrane.org). We calculated the standard mean difference (SMD) with 95\% confidence intervals (CIs). Heterogeneity was assessed using the Cochrane Q-statistic test $\left(\mathrm{P}<0.05\right.$ was considered statistically effective) and the $\mathrm{I}^{2}$-statistic test. A random-effects model would be adopted if $\mathrm{I}^{2}>50 \%$, which indicates substantial heterogeneity. Conversely, a fixed-effects model would be used if $\mathrm{I}^{2}<50 \%$. Sources of heterogeneity were searched as far as possible, and subgroup analysis was carried out when necessary. The sensitivity analysis was performed in order to improve the robustness of the results.

\section{RESULTS}

\section{Study Selection}

For animal studies, a total of 473 potentially relevant hints were identified, of which 401 were duplicated. After screening of the titles and abstracts, seven studies were excluded because of the following reasons: 1) case report; 2) clinical trial; and 3) review article. Then secondary screening was conducted by reading the full text of the remaining 65 studies; 51 studies were excluded because of at least one of the following reasons: 1) failed to obtain full text; 2) non-NGR1 treatment; 3) inappropriate animal model; 4) compared with Chinese herbal medicine or herbal active compounds; 5) no control group; 6) master dissertation or doctoral dissertation; and 7) no available data. Finally, 14 papers (Liu et al., 2010; Deng and Lai, 2013; Han, 2014; He et al., 2014; Li et al., 2014; Meng et al., 2014; Yu et al., 2014; Dong et al., 2015; Xia et al., 2015; Wang et al., 2016; Yu et al., 2016; Zhao et al., 2017; Zou et al., 2017; Tu et al., 2018) were included (Figure 2A).

For cell studies, a total of 112 potentially relevant hints were identified, of which 47 were duplicated. After screening of the titles and abstracts, 13 studies were excluded because of the following reasons: 1) case report; 2) clinical trial; and 3) review article. Then secondary screening was conducted by reading the full text of the remaining 52 studies; 28 studies were excluded because of at least one of the following reasons: 1) failed to obtain full text; 2) nonNGR1 treatment; 3) inappropriate cell model; 4) compared with Chinese herbal medicine or herbal active compounds; 5) no control group; 6) master dissertation or doctoral dissertation; and 7) no 

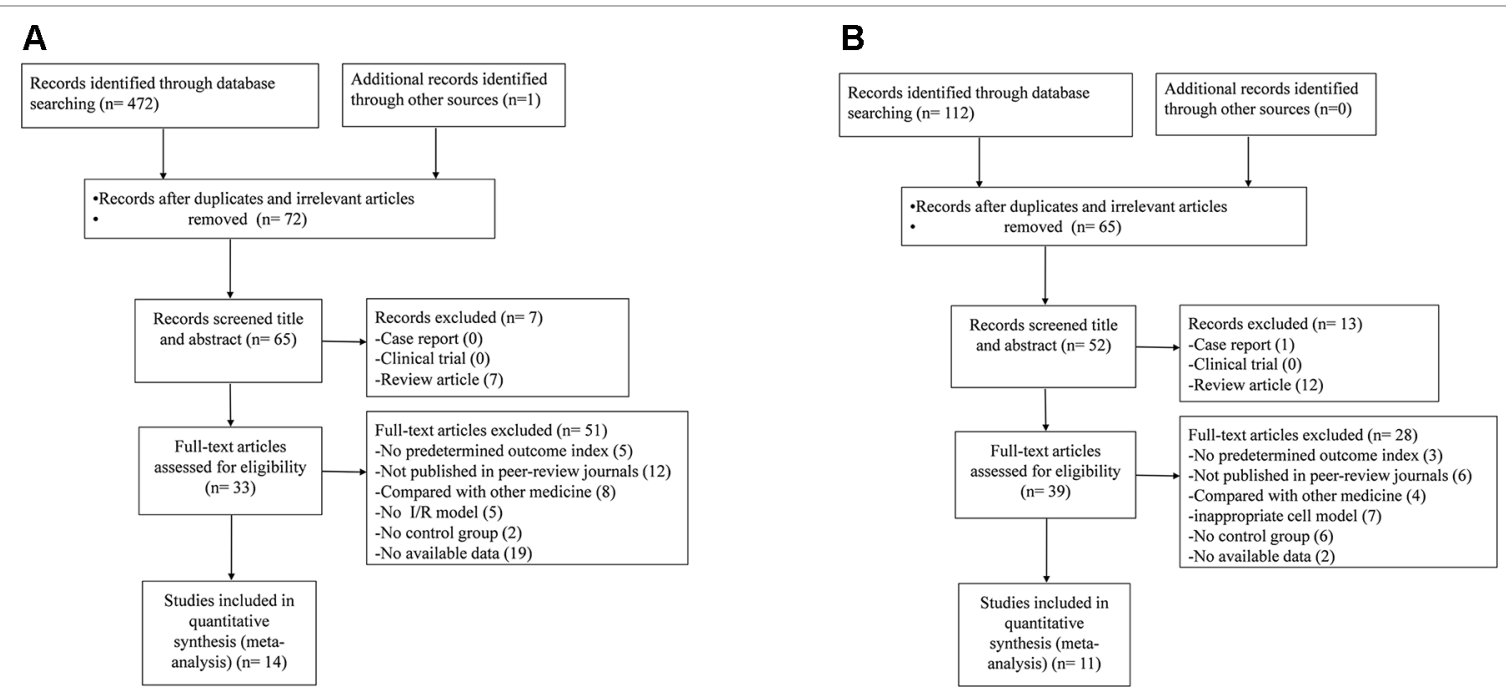

FIGURE 2 | Summary of the process for identifying candidate studies. (A) Search strategy for animal experiments: 473 potentially relevant studies were identified; after removal of duplicates and the application of inclusion and exclusion criteria, 14 studies were included in the meta-analysis. (B) Search strategy for cell experiments: 112 potentially relevant studies were identified; after removal of duplicates and the application of inclusion and exclusion criteria, 11 studies were included in the meta-analysis.

available data. Finally, 11 papers (He et al., 2014; Meng et al., 2014; Wan et al., 2015; Yu et al., 2016; Zhou et al., 2016; Wang et al., 2016; Hou et al., 2017; Wang et al., 2017; Zhou et al., 2017; Liu et al., 2019; Tu et al., 2018) were included (Figure 2B).

\section{Characteristics of Included Studies Animal Experiments}

Fourteen animal experiments between 2010 and 2018 were included. Five studies (Deng and Lai, 2013; Han, 2014; Yu et al., 2014; Dong et al., 2015; Zhao et al., 2017) were published in Chinese, and nine studies (Liu et al., 2010; He et al., 2014; Li et al., 2014; Meng et al., 2014; Xia et al., 2015; Wang et al., 2016; Yu et al., 2016; Zou et al., 2017; Tu et al., 2018) were published in English. Eleven studies (Liu et al., 2010; Deng and Lai, 2013; Han, 2014; He et al., 2014; Li et al., 2014; Meng et al., 2014; Dong et al., 2015; Xia et al., 2015; Yu et al., 2016; Zhao et al., 2017; Zou et al., 2017) used healthy adult Sprague-Dawley (SD) male rats, while one study (Yu et al., 2014) used male Wistar rat and two studies (Wang et al., 2016; Tu et al., 2018) used 7-day-old SD rats. The body weight of adult SD rats ranged from 180 to $300 \mathrm{~g}$. To induce anesthesia, pentobarbital were used in five studies (Liu et al., 2010; He et al., 2014; Yu et al., 2014; Xia et al., 2015; Zhao et al., 2017); chloral hydratein in two studies (Dong et al., 2015; Zhou et al., 2017); isofluranein in two studies (Wang et al., 2016; Tu et al., 2018); urethane in one study (Yu et al., 2016); ulatanin in one study (Han, 2014); ketaminein in one study (Meng et al., 2014); and noanesthetic in one study (Deng and Lai, 2013). To establish animal models of myocardial I/R injury, ligation of left anterior descending coronary artery (LAD) was used in four studies (Han, 2014; He et al., 2014; Yu et al., 2014; Xia et al., 2015); ligation of LAD for $40 \mathrm{~min}$ followed by $60 \mathrm{~min}$ of reperfusion in one study (Yu et al., 2016); ligation of LAD for 30 min followed by 30, 60, and $90 \mathrm{~min}$ of reperfusion in one study (Han, 2014). One study (Deng and Lai, 2013) reported that AMI model induced by injecting pituitrin $(0.65 \mathrm{U} / \mathrm{kg})$ into sublingual. To establish cerebral I/R injury models, occlusion of middle cerebral artery (MCA) was used in three studies (Meng et al., 2014; Dong et al., 2015; Zhao et al., 2017); ligation of common carotid artery (CCL) in two studies (Wang et al., 2016; Tu et al., 2018); and bilateral common carotid artery occlusion (BCCAO) in one study (Zou et al., 2017). One study (Liu et al., 2010) induced renal I/R animal models by clamping left renal artery and vein, and one study (Li et al., 2014) induced intestinal I/R animal models by clamping superior mesenteric artery. For outcome measures, the MI size was used in four studies (Deng and Lai, 2013; Han 2014; He et al., 2014; Xia et al., 2015), serum CK in three studies (Deng and Lai, 2013; Xia et al., 2015; Yu et al., 2016), serum MDA in two studies (Xia et al., 2015; Yu et al., 2016), cerebral infarction volume in five studies (Meng et al., 2014; Dong et al., 2015; Wang et al., 2016; Zou et al., 2017; Tu et al., 2018), neurologic deficit score in two studies (Meng et al., 2014; Dong et al., 2015), serum creatinine in one study (Liu et al., 2010), and Park/Chiu score in one study (Li et al., 2014). The detailed characteristics of the included studies were generalized in Table 1.

\section{Cell Experiments}

Eleven studies involved in cell experiments between 2014 and 2018 were included, of which four studies (Wan et al., 2015; Zhou et al., 2016; Hou et al., 2017; Zhou et al., 2017) were published in Chinese and seven studies (He et al., 2014; Meng et al., 2014; Wang et al., 2016; Yu et al., 2016; Wang et al., 2017; Liu et al., 2019; Tu et al., 2018) in English. Wistar Suckling mice cardiomyocytes were used in three studies (Wan et al., 2015; Zhou et al., 2016; Zhou et al., 2017), and embryonic cardiomyoblast-derived cardiomyocytes (H9C2) of rat were used in two studies (He et al., 2014; Yu et al., 2016). The methods of establishing I/R model in cardiomyocytes 
TABLE 1 | Characteristics of the 14 included animal studies.

\begin{tabular}{|c|c|c|c|c|c|c|c|c|}
\hline $\begin{array}{l}\text { Study } \\
\text { (years) }\end{array}$ & $\begin{array}{l}\text { Species (sex; } \\
\mathrm{n}=\text { experimental/ } \\
\text { control group) }\end{array}$ & Weight & Model(method) & Anesthetic & $\begin{array}{l}\text { Treatment group(Method } \\
\text { to astraddle sides) }\end{array}$ & Control group & Outcome index (time) & Intergroup differences \\
\hline $\begin{array}{l}\text { Deng and } \\
\text { Lai } 2013\end{array}$ & $\begin{array}{l}\text { SD rats (Half } \\
\text { male and female; } \\
\text { 10/10) }\end{array}$ & $180-220 \mathrm{~g}$ & $\begin{array}{l}\text { Sublingual vein } \\
\text { injection of } \\
\text { pituitrin }\end{array}$ & - & $\begin{array}{l}\text { NGR1 }(10 \mathrm{mg} / \mathrm{kg} / \text { day; } \\
\text { i.g.) for } 4 \text { days before } \\
\text { ischemia and } 7 \text { days after } \\
\text { ischemia }\end{array}$ & $\begin{array}{l}\text { Negative control group, } \\
\text { isometric normal saline } \\
\text { (i.g.) for } 4 \text { days before } \\
\text { ischemia and } 7 \text { days } \\
\text { after ischemiaPositive } \\
\text { control group, diltiazem } \\
\text { (i.g.) for } 4 \text { days before } \\
\text { ischemia and } 7 \text { days } \\
\text { after ischemia }\end{array}$ & $\begin{array}{l}\text { 1. ST-segment and inversion rate of T-wave } \\
\text { 2. AST 3. CK 4. CK-MB 5. LDH 6. LDH1 } 7 . \\
\text { Myocardial infarct size 8. Bcl-2 9. Bax }\end{array}$ & $\begin{array}{l}\text { 1. } P<0.012 . P<0.053 . P< \\
0.054 . P<0.055 . P<0.056 . \\
P<0.057 . P<0.058 . P<0.01 \\
\text { 9. } P<0.01\end{array}$ \\
\hline
\end{tabular}

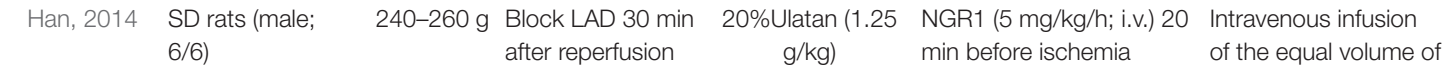

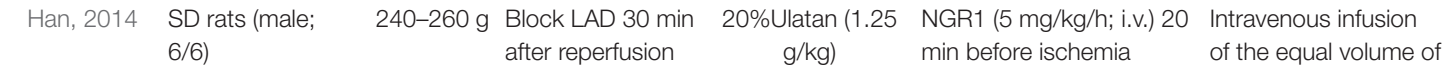

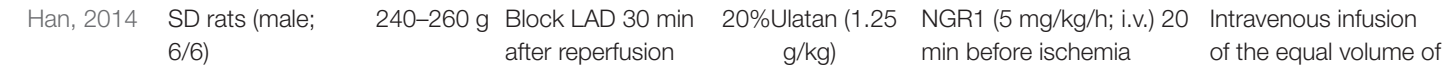

after ischemia

1\% Pentobarbital NGR1 $(2.5 \mathrm{mg} / \mathrm{kg} /$ day; sodium(40 mg/kg) i.p.)for 4 weeks after ischemia

Yu et al., Wistar rats (male; 220-280 g Block LAD 13/13)

He et al., SD rats (male;

2014 8/8)

Xia et al. $\quad$ SD rats (male; 2015 6/6)

$\begin{array}{lll}\text { Yu et al., } \quad \text { SD rats (male; } \quad \text { 200-220 } \mathrm{g} & \text { The isolated } \\ 2016 & \text { Langendorff- } \\ & \text { perfused rat } \\ & \text { hearts received } \\ & \text { ischemia/ } \\ & \text { reperfusion(40 } \\ & \text { min/60 } \mathrm{min})\end{array}$

$2 \%$ after reperfusion sodium (i.v.) for $30 \mathrm{~min}$ before ischemia; and 90 ming ischemia; normal saline 1. Vent leakagr leakage (\%) 3 . Coronary blood flow (\%) 4 IVDP 7. IVSP 8 + +dP/dtmax 9. - dP/d 10. MPO 11 1.P<0.05 2. $P<0.053 . P<$ 6. $P>0.057 . P<0.058 . P<0.05$ 12. CD18 13. Positive 0.05 12. $P<0.0513 . P<0.05$ percent of TUNEL (\%) 14. ATP 15. ADP 16. 14. $P<0.05$ 15. $P>0.05$ 16. $P>$ AMP 17. ADP/ATP 18. AMP/ATP 19. ATP $\alpha / 0.05$ 17. $P<0.05$ 18. $P<0.05$ GAPDH 20. ATP5D/GAPDH 21. ATP $/ \quad 19 . P>0.0520 . P<0.0521 . P>$ GAPDH 22. ZO-1/GAPDH 23. VE/GAPDH $\quad 0.05$ 22. $P<0.05$ 23. $P<0.05$ $\begin{array}{ll}\text { 24. JAM-1/GAPDH 25. Claudin-5/GAPDH } & 24 . P<0.0525 . P<0.0526 . P<\end{array}$ 26. Cav-1/GAPDH 27. Cav-3/GAPDH 28.p- 0.05 27. $P<0.05$ 28. $P<0.05$ Src/Src 29.Src/GAPDH

Intraperitoneal injection 1. MVC 2. MVD 3. VEGF 4. bFGF $\quad$ 1. P $<0.052 . P<0.053 . P<$ of equal volume of saline after ischemia

Continuous injection of 1. AAR/LV 2. Myocardial infarct size/AAR saline $(1 \mathrm{~m} / \mathrm{h})$ 3.+dP/dtmax 4.- dP/dtmax 5. LVSP 6. $0.054 . P<050$

1. $P>0.05$ 2. $P<0.05$ 3. $P>$ 0.05 4. $P<0.05$ 5. $P>0.056$ $\begin{array}{ll} & \\ & \end{array}>0.057 . P<0.058 . P<0.05$ Cleaved caspase-3/procaspase-3 10. ATP $9 . P<0.05$ 10. $P<0.05$ 11. $P>$ 11. AMP 12. P-AMPK/ $\beta$-actin 13. ATP $\quad 0.0512 . P<0.0513 . P>0.05$ synthase- $\alpha / \beta$-actin 14. ATP synthase- $\beta / \beta-\quad 14 . P>0.0515 . P<0.0516$. $P<$ actin 15. ATP $5 D / \beta$-actin 16. ROCK/ $\beta$-actin 0.05 17. $P<0.05$

17. P-MYPT1/MYPT1

1. Myocardial infarct size 2. CK 3. LDH 4. 1. $P<0.012$. $P<0.013$. $P<0.05$ T-SOD 5. MDA 6. IL-1 $\beta$ 7. IL-8 8. TNF- $\alpha$ 9. $\quad$ 4. $P<0.001$ 5. $P<0.01$ 6. $P<$ p-NF-кBP65/NF-кBP65 10. p-|кB $\alpha / 1 \kappa B \alpha \quad 0.017 . P<0.018 . P<0.019 . P<$ 11. VDUP1/GAPDH

1. LVSP 2. Heart rate 3. $+\mathrm{dp} / \mathrm{dtmax} 4$. -dp/dt min 5. MDA 6. SOD 7. CAT 8. CK 9. GSH-Px activities 10. P-JNK/JNK 11. $\mathrm{CHOP} / \beta$-actin 12. Bcl-2/BAX 13. GRP78/ $\beta$-actin 14. P-PERK/PERK 15. P-elf2 $\alpha /$ elf2 $\alpha$ 16. IRE $1 / \beta$-actin 17. ATF6/ $\beta$-actin 18 . Caspase-12/ $\beta$-actin 19. BAX/ $\beta$-actin 


\begin{tabular}{|c|c|c|c|c|c|c|}
\hline $\begin{array}{l}\text { Study } \\
\text { (years) }\end{array}$ & $\begin{array}{l}\text { Species (sex; } \mathbf{n}= \\
\text { experimental/ } \\
\text { control group) }\end{array}$ & Weight & Model(method) & Anesthetic & $\begin{array}{l}\text { Treatment group(Method } \\
\text { to astraddle sides) }\end{array}$ & Control group \\
\hline $\begin{array}{l}\text { Meng et al., } \\
2014\end{array}$ & $\begin{array}{l}\text { SD rats (male; } \\
40 / 40 \text { ) }\end{array}$ & $250-300 \mathrm{~g}$ & MCAO & $\begin{array}{l}\text { Ketamine ( } 80 \text { mg/ } \\
\text { kg; i.p.) }\end{array}$ & $\begin{array}{l}\text { NGR1 (20 mg/kg; i.p) } \\
\text { before ischemia }\end{array}$ & $\begin{array}{l}\text { Given the same } \\
\text { amount of saline }\end{array}$ \\
\hline
\end{tabular}

Dong SD rats (male; $\quad 180-200 \mathrm{~g}$ MCAO

et al., 2015 8/8)

$\begin{array}{llll}\text { Wang et al., } & \text { 7-day-old SD rats } & - & \text { The common } \\ 2016 & \text { (male; 5/5) } & & \text { carotid artery }\end{array}$

Zhao et al., SD rats (male;

2017 10/10)

Zou et al., SD rats(male;

$2017 \quad$ 15/15

Tu et al., 7-day-old SD rats _ _ CCL

2018 (male; 9/9)
10\% Chloral NGR1 (7.0 mg/kg; i.p.) for Given the same hydrate $(300 \mathrm{mg} / 14$ days after ischemia amount of saline kg; i.p.)

Isoflurane (2.5\%) NGR1 (15 mg/kg. $12 \mathrm{~h}$; i.p.) after CCL; before exposure to the hypoxic environment

3\% Pelltobarbitalum NGR1 ( $5 \mathrm{mg} / \mathrm{ml}$; i.v.) for Natricum $(0.2 \quad 3$ days $\mathrm{m} / 100 \mathrm{~g})$

Chloral hydrate NGR1 (100 mg/kg; i.g.) (350 mg/kg; i.p.) after ischemia

(ischemia; 20 min reperfusion; $3 \mathrm{~h}$ )

Isoflurane (2.5\%) NGR1 (15 mg/kg; i.p.; $\mathrm{q} 12 \mathrm{~h}$ ) for 2 days after ischemia

Given the same amount of saline

Intragastric $\mathrm{ml}$ saline

Not mentioned
1. Infarction volumes 2. Neurologic deficit score 3. TUNEL-positive cells rate 4. Caspase-3 activity 5. NADPH oxidase activity 6 . Superoxide levels 7 Mitochondrial superoxide levels 8. MDA 9. Protein carbonyl levels 10. 8-OHdG levels

11. HO-1 activity

1. Infarction volumes 2. Neurologic deficit $\quad$ 1. $P<0.012$ 2. $P<0.013$. $P<$ score 3. Population spike 4. Escape latency 5. Target quadrant dwell time

1. Infarction volumes 2. Ratio of GRP78/ $\beta$ - $\quad$ 1. $P<0.052$ 2. $P<0.05$ 3. $P<$ actin 3. Ratio of P-PERK/PERK 4. Ratio of 0.05 4. $P<0.055$. $P<0.05$

P-IRE1 $\alpha / \operatorname{IRE} 1 \alpha$ 5. Ratio of $\mathrm{CHOP} / \beta$-actin

1. The number of TUNEL-positive cells 2. 1. $P<0.052$. $P<0.05$ TNF- $\alpha$ mRNA

1. Cerebral infarction size 2. Relative expression 1. $P<0.012$ 2. $P<0.013$. $P<$ of BDNF mRNA 3. Relative expression of $\quad 0.014 . P<0.01$

$\mathrm{BCl}-2$ to $\beta$-actin 4. Relative expression of $\mathrm{Bax}$ to $\beta$-actin positive nuclei 4 . Brain weight ratio 5 . The score of balance beam 6 . Percent in the target quadrant 7. PI3K/ $\beta$-actin 8. P-Akt/T- $0.0512 . P<0.0113 . P<0.05$ Akt 9. P-mTOR/T-mTOR 10. P-P70S6K P70S6K 11. P-4EBP-1/4EBP-1 12.

P-JNK/T-JNK 13. P-C-JUN/C-JUN

\begin{tabular}{|c|c|c|c|c|c|c|c|c|}
\hline $\begin{array}{l}\text { Liu et al., } \\
2010\end{array}$ & $\begin{array}{l}\text { SD rats (male; } \\
6 / 6 \text { ) }\end{array}$ & $230-250 \mathrm{~g}$ & $\begin{array}{l}\text { Clamping left renal } \\
\text { arteryand vein } \\
\text { (ischemia; } 45 \mathrm{~min} \text {; }\end{array}$ & $\begin{array}{l}\text { Pentobarbital } \\
\text { sodium (50 mg/ } \\
\text { kg) }\end{array}$ & $\begin{array}{l}\text { NGR1 ( } 40 \mathrm{mg} / \mathrm{kg} \text {; i.p.) } \\
\text { before ischemia and for } 3 \\
\text { days after reperfusion }\end{array}$ & $\begin{array}{l}\text { Receiving the same } \\
\text { amount of saline }\end{array}$ & $\begin{array}{l}\text { 1. Serum levels of creatinine } 2 \text {. MPO } 3 \text {. } \\
\text { Relative TNF- } \alpha \text { band intensity } 4 \text {. TUNEL- } \\
\text { positive cells } 5 \text {. Relative p38MAPK band }\end{array}$ & $\begin{array}{l}\text { 1. } P<0.052 . P<0.053 . P< \\
0.054 . P<0.055 . P<0.056 . \\
P<0.05\end{array}$ \\
\hline $\begin{array}{l}\text { Li et al., } \\
2014\end{array}$ & $\begin{array}{l}\text { SD rats (male; } \\
6 / 6 \text { ) }\end{array}$ & $200-220 \mathrm{~g}$ & $\begin{array}{l}\text { Clamping superior } \\
\text { mesenteric artery } \\
(90 \mathrm{~min} / 1 \text { or } 72 \mathrm{~h})\end{array}$ & $\begin{array}{l}\text { Pentobarbital } \\
\text { sodium ( } 50 \mathrm{mg} / \\
\mathrm{kg} \text { ) }\end{array}$ & $\begin{array}{l}\text { NGR1 }(10 \mathrm{mg} / \mathrm{kg} / \mathrm{h} \text {; } \\
\text { IVgtt) for } 170 \mathrm{~min} \text { after } \\
\text { reperfusion }\end{array}$ & $\begin{array}{l}\text { Receiving the same } \\
\text { amount of saline }\end{array}$ & $\begin{array}{l}\text { 1. IkB- } \alpha \text { change (\%) 2. NF-kB change (\%) } \\
\text { 3. ATP5D change (\%) 4. Zonulaoccludens } \\
-1 \text { change (\%) 5. Occludin change (\%) } 6 \text {. } \\
\text { Claudin- } 5 \text { change } \% \text { ) }\end{array}$ & $\begin{array}{l}\text { 1. } P<0.052 . P<0.053 . P< \\
0.054 . P<0.055 . P<0.056 . \\
P<0.05\end{array}$ \\
\hline
\end{tabular}

SD rats, Spraque-Dawley rats; LAD, the left anterior descending coronary artery; SOD, superoxide dismutase; MDA, malondialdehyde; CK, creatine kinase; LDH, lactate dehydrogenase; CK-MB, creatine kinase-MB; TNF- $\alpha$, tumor necrosis factor- $\alpha$; GSH-Px, glutathione peroxidase; CAT, catalase; AST, aspartate aminotransferase; AAR/LV, area at risk/left ventricle; +dp/dtmax, maximum ascending rate of left ventricular pressure; -dP/dtmax, maximum descending rate of left ventricular pressure; LVSP, left ventricular systolic pressure; LVDP, left ventricular diastolic pressure; MVC, miniature blood vessel; MVD, microvascular density; VEGF, vascular endothelial growth factor; bFGF, basic fibroblast growth factor; ATP, adenosine triphosphate; AMP, adenosine monophosphate; LL-1, interleukin-1; IL-8, interleukin-8; NF-kBp65, nuclear factor-kappa Bp65; AMPK, AMP-activated protein kinase; ROCK, Rho-associated coil kinase; MYPT1, myosin phosphatase target subunit-1; NF-кBP65, nuclear factor-kBp65; /KBa, nuclear factor of kappa light polypeptide gene enhancer in B-cells inhibitor; alpha; VDUP1, vitamin D3 upregulated protein 1; GAPDH, glyceraldehyde-3-phosphate dehydrogenase; c-JNK, c-Jun N-terminal kinase; BCI-2, B-cell lymphoma-2; MCAO; middle cerebral artery occlusion; BCCAO, bilateral common carotid artery occlusion; BDNF, brain-derived neurotrophic factor; NGR1, notoginsenoside R1; AMI, acute myocardial ischemia; bax, Bcl-2associated X protein; RBC, red blood cell; MPO, myeloperoxidase; ICAM-1, intercellular cell adhesion molecule-1; TUNEL, TdT-mediated dUTP nick-end labeling; ADP, adenosine diphosphate; GAPDH, glyceraldehyde-3-phosphate dehydrogenase; ZO-1, Zonula occludens-1; JAM-1, recombinant junctional adhesion molecule 1; Cav-1, caveolin 1; Cav-3, caveolin 3; AAR, area at risk; T-SOD, total superoxide dismutase; LL-13, interleukin 1 beta; CHOP, C/EBP homologous protein; GRP78, glucose regulated protein 78; PERK, protein kinase R-like ER kinase; $p$-PERK, phospho-protein kinase R-like ER kinase; elf2 $\alpha$, eukaryotic initiation factor 2 20; IRE1, inositol-requiring enzyme-1 1 ; ATF6, activating transcription factor 6; 8-OHdG, 8-hydroxydeoxyguanosine; HO-1, heme oxygenase-1; PI3K, p-mTOR, phospho-mammalian target of rapamycin; T-mTOR, P70S6K, protein S6 kinase; P-P70S6K, phospho-protein S6 kinase; P-4EBP-1, phospho-4EBP1. 
include the application of hydrogen peroxide $\left(\mathrm{H}_{2} \mathrm{O}_{2}\right)$ (Wan et al., 2015; Zhou et al., 2016; Zhou et al., 2017) and OGD/R (He et al., 2014; Yu et al., 2016). The dosages of NGR1 were 10 $\mu \mathrm{mol} / \mathrm{L}$ in two studies (Zhou et al., 2016; Zhou et al., 2017), $20 \mu \mathrm{mol} / \mathrm{L}$ in one study (Yu et al., 2016), and $100 \mu \mathrm{mol} / \mathrm{L}$ in two studies (He et al., 2014; Wan et al., 2015). Cell viability and TUNEL-positive rate were used as outcome measures in six studies (He et al., 2014; Wan et al., 2015; Zhou et al., 2016; Yu et al., 2016; Zhou et al., 2017; Liu et al., 2019), LDH in seven studies (He et al., 2014; Meng et al., 2014; Wang et al., 2016; Zhou et al., 2016; Zhou et al., 2017; Hou et al., 2017; Tu et al., 2018), SOD in three studies (Wan et al., 2015; Zhou et al., 2016; Zhou et al., 2017), and MDA in four studies (Meng et al., 2014; Wan et al., 2015; Zhou et al., 2016; Zhou et al., 2017). The detailed characteristics of the included studies were generalized in Table 2 .

In addition, seven studies (Liu et al., 2010; Meng et al., 2014; Yu et al., 2014; Xia et al., 2015; Yu et al., 2016; Zou et al., 2017; Tu et al., 2018) reported chemical analysis of NGR1 in animal studies. Seven studies (Meng et al., 2014; Wan et al., 2015; Wang et al., 2016; Wang et al., 2016; Liu et al., 2019; Tu et al., 2018) reported chemical analysis of NGR1 in cell studies. The characteristics of NGR1 were shown in Table 3.

\section{Study Quality \\ Animal Studies}

The scores of study quality ranged from 3 to 8 in a total of 10 points. All included studies were peer-reviewed publication. All the studies reported that the animals were allocated randomly to treatment or control group, and the anesthetics used in the experiments with no intrinsic organ-protective activity. However, sample size calculation, blinded induction of model, and blinded assessment of outcome were not reported in all included studies. Seven studies (He et al., 2014; Meng et al., 2014; Xia et al., 2015; Wang et al., 2016; Yu et al., 2016; Zou et al., 2017; Tu et al., 2018) stated compliance with animal welfare regulations and eight studies (He et al., 2014; Meng et al., 2014; Yu et al., 2014; Dong et al., 2015; Xia et al., 2015; Wang et al., 2016; Yu et al., 2016; Tu et al., 2018) declared no potential conflict of interests. The methodological quality of included studies was shown in Table 4.

\section{Cell Studies}

The scores of study quality ranged from 3 to 5 in a total of 10 points. All included studies were peer-reviewed publication. All the studies reported control of experimental conditions, the effect or safety of treatment, and statement of no potential conflict of interests. Nine studies (Meng et al., 2014; Wan et al., 2015; Wang et al., 2016; Zhou et al., 2016; Hou et al., 2017; Wang et al., 2017; Zhou et al., 2017; Liu et al., 2019; Tu et al., 2018) used primary cultured cells and two studies (He et al., 2014; Yu et al., 2016) used subcultured cells. However, sample size calculation and blinded assessment of outcome were not reported in all included studies. The methodological quality was shown in Table 5.

\section{Effectiveness Myocardial I/R Injury MI Size}

Meta-analysis of four studies (Deng and Lai, 2013; Han, 2014; He et al., 2014; Xia et al., 2015) showed NGR1 had significant effect on reducing MI size compared with control group [ $=60$, SMD: $-2.01,95 \%$ CI: -2.67 to $-1.35, \mathrm{P}<0.00001$; heterogeneity $\chi^{2}=1.24$, $\left.\mathrm{df}=3(\mathrm{P}=0.74), \mathrm{I}^{2}=0 \%\right]$ (Figure 3A).

\section{Cardiac Enzyme}

Meta-analysis of three studies (Deng and Lai, 2013; Xia et al., 2015; Yu et al., 2016) showed NGR1 had significant effect on decreasing CK compared with the control group [ $n=52$, SMD: $-5.05,95 \%$ CI: -8.83 to $-1.28, P=0.009$; heterogeneity: $\chi^{2}=22.94$, $\mathrm{df}=2$ $\left.(P<0.0001), \mathrm{I}^{2}=91 \%\right]$. Owing to the obvious heterogeneity, we conducted a sensitivity analyses and removed one study (Yu et al., 2016) that utilized Langendroff-perfused rat hearts. Metaanalysis of the remaining two studies (Deng and Lai, 2013; Xia et al., 2015) showed NGR1 had significant effect on decreasing CK compared with the control group $[n=32$, SMD: $-2.06,95 \%$ CI: -2.96 to $-1.15, P<0.00001$; heterogeneity: $\chi^{2}=0.02, \mathrm{df}=1$ $\left.(P=0.89), \mathrm{I}^{2}=0 \%\right]$ (Figure $\left.3 \mathrm{~B}\right)$. We failed to conduct metaanalysis of serum MDA in the two studies (Xia et al., 2015; Yu et al., 2016) because of high heterogeneity. However, both of them favored that NGR1 treatment could reduce the level of serum MDA compared with the control group $(P<0.05)$.

\section{Cardiomyocyte Apoptosis Rate}

Meta-analysis of six studies (He et al., 2014; Wan et al., 2015; Yu et al., 2016; Zhou et al., 2016; Zhou et al., 2017; Liu et al., 2019) showed NGR1 had significant effect on reducing TUNEL-positive cell rate compared with the control group [ $n=72$, SMD: -10.94 , 95\% CI: -14.77 to $-7.11, P<0.00001$; heterogeneity: $\chi^{2}=13.83$, $\left.\mathrm{df}=5(P=0.02), \mathrm{I}^{2}=64 \%\right]$. Owing to the obvious heterogeneity, we conducted a sensitivity analyses and removed two studies (He et al., 2014; Yu et al., 2016) that utilized subcultured cells. Meta-analysis of the remaining four studies (Wan et al., 2015; Zhou et al., 2016; Zhou et al., 2017; Liu et al., 2019) showed NGR1 had significant effect on decreasing TUNEL-positive cell rate compared with the control group [ $n=48$, SMD: $-9.51,95 \%$ CI: -12.80 to $-6.23, P<0.00001$; heterogeneity: $\chi^{2}=5.27, \mathrm{df}=3$ $\left.(P=0.15), \mathrm{I}^{2}=43 \%\right]$ (Figure 3C).

\section{Cardiomyocyte Viability}

Meta-analysis of six studies (He et al., 2014; Wan et al., 2015; Yu et al., 2016; Zhou et al., 2016; Zhou et al., 2017; Liu et al., 2019) showed NGR1 had significant effect on increasing cell viability compared with the control group [ $n=36$, SMD: 9.31, 95\% CI: 7.21 to $11.41, P<0.00001$; heterogeneity: $\chi^{2}=5.65, \mathrm{df}=5(P=$ $\left.0.34), \mathrm{I}^{2}=12 \%\right]$ (Figure 4).

\section{Cardiomyocytes $\mathrm{LDH}$}

Meta-analysis of four studies (He et al., 2014; Yu et al., 2016; Zhou et al., 2016; Zhou et al., 2017) showed NGR1 had significant effect on decreasing cell LDH compared with the control group [ $n=48$, SMD: $-13.57,95 \% \mathrm{CI}:-21.27$ to $-5.88, P=0.0005$; 
TABLE 2 | Characteristics of the 11 included cell studies.

\begin{tabular}{|c|c|c|c|c|c|c|c|c|}
\hline Study (years) & $\begin{array}{l}\text { Appellation }(\mathbf{n}= \\
\text { experimental/ } \\
\text { control group) }\end{array}$ & $\begin{array}{l}\text { Organism age } \\
\text { tissue }\end{array}$ & $\begin{array}{l}\text { Primary } \\
\text { cells or } \\
\text { subcultured } \\
\text { cells }\end{array}$ & $\begin{array}{l}\text { Model } \\
\text { (method) }\end{array}$ & $\begin{array}{l}\text { Treatment } \\
\text { group (method } \\
\text { to astragal } \\
\text { sides) }\end{array}$ & $\begin{array}{l}\text { Control } \\
\text { group }\end{array}$ & Outcome index (time) & Intergroup differences \\
\hline $\begin{array}{l}\text { Zhou et al., } \\
2016\end{array}$ & $\mathrm{RCM}(6 / 6)$ & $\begin{array}{l}\text { WistarSuckling } \\
\text { miceMyocardium }\end{array}$ & Primary cells & $\begin{array}{l}\text { Received } \\
\mathrm{H}_{2} \mathrm{O}_{2} \\
(50 \mu \mathrm{mol} / \mathrm{L})\end{array}$ & $\begin{array}{l}\text { NGR1 }(10 \\
\mu m o l / L ; 24 \mathrm{~h}) \\
\text { before molding }\end{array}$ & No treatment & $\begin{array}{l}\text { 1. LDH 2. SOD 3. MDA 4. Cell viability } 5 . \\
\text { Apoptosis rate 6. p-ERK1/2 7. ERK1/2 } 8 . \\
\text { p-p38 9. p38 }\end{array}$ & $\begin{array}{l}\text { 1. } P<0.012 . P<0.013 . P< \\
0.014 . P<0.015 . P<0.016 . \\
P<0.017 . P<0.018 . P<0.01 \\
9 . P<0.01\end{array}$ \\
\hline $\begin{array}{l}\text { Zhou et al., } \\
2017\end{array}$ & $\mathrm{RCM}(6 / 6)$ & $\begin{array}{l}\text { WistarSuckling } \\
\text { miceMyocardium }\end{array}$ & Primary cells & $\begin{array}{l}\text { Received } \\
\mathrm{H}_{2} \mathrm{O}_{2} \\
(50 \mu \mathrm{mol} / \mathrm{L})\end{array}$ & $\begin{array}{l}\text { NGR1 }(10 \\
\text { mol/L; } 24 \mathrm{~h}) \\
\text { before molding }\end{array}$ & No treatment & $\begin{array}{l}\text { 1. LDH 2. SOD 3. MDA 4. Cell viability } 5 . \\
\text { Apoptosis rate }\end{array}$ & $\begin{array}{l}\text { 1. } P<0.01 \text { 2. } P<0.013 . P<0.01 \\
\text { 4. } P<0.015 . P<0.01\end{array}$ \\
\hline $\begin{array}{l}\text { Wan et al., } \\
2015\end{array}$ & $\operatorname{RCM}(6 / 6)$ & $\begin{array}{l}\text { WistarSuckling } \\
\text { miceMyocardium }\end{array}$ & Primary cells & $\begin{array}{l}\text { Received } \\
\mathrm{H}_{2} \mathrm{O}_{2} \\
(1 \mathrm{mmol} / \mathrm{L})\end{array}$ & $\begin{array}{l}\text { Received NGR1 } \\
\text { (100 } \mu \mathrm{mol} / \mathrm{L} ; 24 \\
\text { h) before molding }\end{array}$ & No treatment & $\begin{array}{l}\text { 1. Cell viability 2. Apoptosis rate 3. MDA } 4 . \\
\text { soD5. p-JNK 6. Bax } 7 . \text { Bcl -2 }\end{array}$ & $\begin{array}{l}\text { 1. } P<0.05 \text { 2. } P<0.05 \text { 3. } P<0.05 \\
\text { 4. } P<0.05 \text { 5. } P<0.056 . P<0.05 \\
\text { 7. } P<0.05\end{array}$ \\
\hline Yu et al., 2016 & H9C2 (6/6) & $\begin{array}{l}\text { Rat embryonic } \\
\text { cardiomyoblast- } \\
\text { derived H9c2 } \\
\text { cardiomyocytes }\end{array}$ & $\begin{array}{l}\text { Subcultured } \\
\text { cells }\end{array}$ & $\begin{array}{l}\mathrm{H} / \mathrm{R} \\
(6 \mathrm{~h} / 12 \mathrm{~h})\end{array}$ & $\begin{array}{l}\text { NGR1 }(20 \\
\mu m o / / L ; 24 \mathrm{~h}) \\
\text { before molding }\end{array}$ & No treatment & $\begin{array}{l}\text { 1. Cell viability2. Extracellular LDH3. } \\
\text { ROS4. Relative intensity of red/green } \\
\text { fluorescence5. PIP positive cell rate6. } \\
\text { TUNEL-positive7. GRP78/ } \beta \text {-actin8. } \\
\text { P-PERK/PERK9. P- elf2 } \alpha / \text { elf2 } \alpha 10 \text {. IRE1/ } \beta \text { - } \\
\text { actin11. ATF6/ } \beta \text {-actin }\end{array}$ & $\begin{array}{l}\text { 1. } P<0.0012 . P<0.0013 . P< \\
0.014 . P<0.0015 . P<0.016 . \\
P<0.0017 . P<0.018 . P<0.001 \\
9 . P<0.00110 . P<0.0111 . P< \\
0.001\end{array}$ \\
\hline He et al., 2014 & H9C2 (6/6) & $\begin{array}{l}\text { A rat cardiac myoblast } \\
\text { cell line }\end{array}$ & $\begin{array}{l}\text { Subcultured } \\
\text { cells }\end{array}$ & $\begin{array}{l}\mathrm{OGD} / \mathrm{R}(15 \\
\text { h) }\end{array}$ & $\begin{array}{l}\text { NGR1 } \\
(100 \mu \mathrm{mol} / \mathrm{L})\end{array}$ & No treatment & $\begin{array}{l}\text { 1. TUNEL-positive2. Cell viability3. } \\
\text { LDH4. Bcl-2/Bax5. Cleaved caspase-3/ } \\
\text { procaspase-36. ATP7. AMP8. ATP } \\
\text { synthase activity9. P-AMPK/ } \beta \text {-actin10. } \\
\text { ATPsynthase- } \alpha / \beta \text {-actin11. ATP synthase- } \\
\beta / \beta \text {-actin12. ATP 5D/ } \beta \text {-actin13. ROCK/ } \beta \text { - } \\
\text { actin14. P-MYPT1/MYPT1 }\end{array}$ & $\begin{array}{l}\text { 1. } P<0.05 \text { 2. } P<0.05 \text { 3. } P<0.05 \\
\text { 4. } P<0.05 \text { 5. } P<0.056 . P<0.05 \\
\text { 7. } P<0.05 \text { 8. } P<0.059 . P<0.05 \\
\text { 10. } P>0.0511 . P>0.0512 . P< \\
0.05 \text { 13. } P<0.0514 . P<0.05\end{array}$ \\
\hline Liu et al., 2019 & $\mathrm{RCM}$ & $\begin{array}{l}\text { NeonatalSD rats } \\
\text { Myocardium }\end{array}$ & Primary cells & OGD (6 h) & $\begin{array}{l}\text { NGR1 ( } 20 \\
\mu \mathrm{mol} / \mathrm{L}) \text { for } 24 \mathrm{~h}\end{array}$ & No treatment & $\begin{array}{l}\text { 1. Cell viability2. Apoptotic cells3. RNA level } \\
\text { expression of miR-214. mRNA and protein } \\
\text { levels of PTEN }\end{array}$ & $\begin{array}{l}\text { 1. } P<0.05 \text { 2. } P<0.053 . P<0.05 \\
P<0.05\end{array}$ \\
\hline $\begin{array}{l}\text { Meng et al., } \\
2014\end{array}$ & $\begin{array}{l}\text { Primary cortical } \\
\text { neurons }(6 / 6)\end{array}$ & $\begin{array}{l}\mathrm{SD} \text { rats embryo } \\
\text { cerebral cortices }\end{array}$ & Primary cells & $\begin{array}{l}\mathrm{OGD} / \mathrm{R}(2 \\
\mathrm{h} / 24 \mathrm{~h})\end{array}$ & $\begin{array}{l}\text { NGR1 }(25 \mu \mathrm{M}) \\
\text { for } 24 \mathrm{~h} \text { before } \\
\text { ischemia }\end{array}$ & $\begin{array}{l}\text { Treated with } \\
\text { DMSO (final } \\
\text { concentration } \\
\text { was } 0.1 \% \text { ) }\end{array}$ & $\begin{array}{l}\text { 1. Intracellular ROS2. NADPH } \\
\text { oxidase activity3. Superoxide levels4. } \\
\text { Mitochondrial superoxide5. MDA6. } \\
\text { Protein carbonyl7. 8-OHdG8. TUNEL- } \\
\text { positive cells rate9. Apoptosis rate10. } \\
\text { Ratio of red to green fluorescence } \\
\text { intensity11.Cell viability12. LDH13. } \\
\text { Caspase-3 activity }\end{array}$ & $\begin{array}{l}\text { 1. } P<0.01 \text { 2. } P<0.01 \text { 3. } P<0.01 \\
\text { 4. } P<0.01 \text { 5. } P<0.016 . P<0.01 \\
\text { 7. } P<0.01 \text { 8. } P<0.019 . P<0.01 \\
\text { 10. } P<0.0111 . P<0.0112 . P< \\
0.0113 . P<0.01\end{array}$ \\
\hline Wang, 2016 & $\begin{array}{l}\text { Primary cortical } \\
\text { neurons }(5 / 5)\end{array}$ & $\begin{array}{l}\text { SD rats embryo } \\
\text { cerebral cortices }\end{array}$ & Primary cells & $\begin{array}{l}\text { OGD/R (1.5 } \\
h / 24 h)\end{array}$ & $\begin{array}{l}\text { NGR1 (10 } \\
\mu \mathrm{mol} / \mathrm{L})\end{array}$ & DMSO (1\%) & 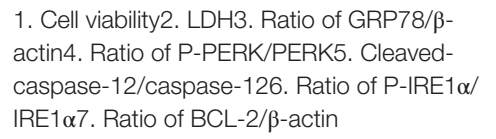 & $\begin{array}{l}\text { 1. } P<0.05 \text { 2. } P<0.053 . P<0.05 \\
\text { 4. } P<0.05 \text { 5. } P<0.056 . P<0.05 \\
\text { 7. } P<0.05\end{array}$ \\
\hline $\begin{array}{l}\text { Hou et al., } \\
2017\end{array}$ & $\begin{array}{l}\text { Primary cortical } \\
\text { neurons }(5 / 5)\end{array}$ & $\begin{array}{l}\text { SD rats embryo } \\
\text { cerebral cortices }\end{array}$ & Primary cells & $\begin{array}{l}\text { OGD/R (1.5 } \\
h / 24 h)\end{array}$ & $\begin{array}{l}\text { NGR1 (10 } \\
\mu \mathrm{mol} / \mathrm{L})\end{array}$ & & $\begin{array}{l}\text { 1. Cell viability2. LDH3. ATF6/Akt4. P-Akt/ } \\
\text { Akt5. Cleaved Caspase-3/ } \beta \text {-actin6. } \\
\text { Bax/ } \beta \text {-actin }\end{array}$ & $\begin{array}{l}\text { 1. } P<0.05 \text { 2. } P<0.05 \text { 3. } P<0.05 \\
\text { 4. } P<0.05 \text { 5. } P<0.056 . P<0.05\end{array}$ \\
\hline
\end{tabular}




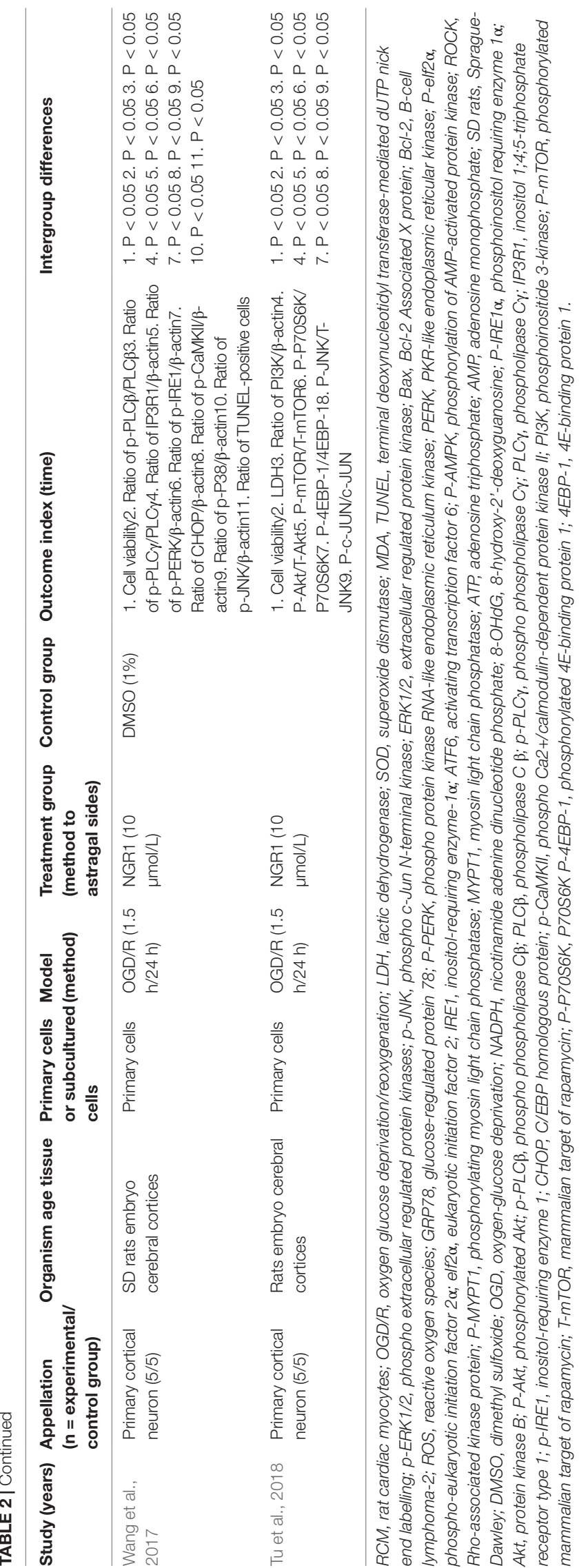

heterogeneity: $\left.\chi^{2}=16.3, \mathrm{df}=3(P=0.001), \mathrm{I}^{2}=82 \%\right]$. Owing to high heterogeneity, we conducted a sensitivity analyses and removed one study (He et al., 2014) for non-pretreatment with NGR1. Meta-analysis of the remaining three studies (Yu et al., 2016; Zhou et al., 2016; Zhou et al., 2017) showed that NGR1 had significant effect on reducing cell LDH compared with the control group [ $n=36$, SMD: $-16.22,95 \%$ CI: -20.93 to -11.51 , $P<0.00001$; heterogeneity: $\left.\chi^{2}=1.92, \mathrm{df}=2(P=0.38), \mathrm{I}^{2}=0 \%\right]$ (Figure 5).t

\section{Cerebral Injury}

\section{Cerebral Infarction Volume}

Meta-analysis of five studies (Meng et al., 2014; Dong et al., 2015; Wang et al., 2016; Zou et al., 2017; Tu et al., 2018) demonstrated NGR1 had significant effect on decreasing cerebral infarction volume compared with the control group [ $n=94$, SMD: -5.25 , 95\% CI: -6.24 to $-4.27, P<0.00001$; heterogeneity: $\chi^{2}=13.65$, $\mathrm{df}=$ $\left.4(P=0.009), \mathrm{I}^{2}=71 \%\right]$. Owing to the obvious heterogeneity, we conducted a sensitivity analyses and removed two studies (Wang et al., 2016; Tu et al., 2018) that utilized 7-day-old SD rats. Metaanalysis of the remaining three studies (Meng et al., 2014; Dong et al., 2015; Zou et al., 2017) demonstrated NGR1 had significant effect on reducing cerebral infarction volume compared with the control group [ $n=66$, SMD: $-6.43,95 \%$ CI: -7.76 to $-5.11, P<$ 0.00001; heterogeneity: $\left.\chi^{2}=3.06, \mathrm{df}=2(\mathrm{P}=0.22), \mathrm{I}^{2}=35 \%\right]$ (Figure 6).

\section{Neurologic Deficit Score}

Meta-analysis of two studies (Meng et al., 2014; Dong et al., 2015) demonstrated NGR1 had significant effect on reducing neurologic deficit score compared with the control group $[n=26$, SMD: $-1.58,95 \%$ CI: -1.87 to $-1.28, P<0.00001$; heterogeneity: $\left.\chi^{2}=0.35, \mathrm{df}=1(P=0.56), \mathrm{I}^{2}=0 \%\right]$ (Figure 7).

\section{Cerebral Cell Viability}

Meta-analysis of five studies (Wang et al., 2016; Wang et al., 2017; Hou et al., 2017; Tu et al., 2018) demonstrated NGR1 had significant effect on increasing cell viability compared with the control group [ $n=54$, SMD: $4.34,95 \%$ CI: 3.16 to 5.52 , $\mathrm{P}<0.00001$; heterogeneity: $\left.\chi^{2}=2.19, \mathrm{df}=4(\mathrm{P}=0.70), \mathrm{I}^{2}=0 \%\right]$ (Figure 8).

\section{Renal Injury}

One study (Liu et al., 2010) reported that NGR1 had significant effect on reducing MPO and serum creatinine compared with the control group $(\mathrm{P}<0.05)$.

\section{Intestinal Injury}

One study (Li et al., 2014) reported that NGR1 had significant effect on reducing MPO and Park/Chiu score compared with the control group $(\mathrm{P}<0.05)$.

\section{Organ-Protection Mechanisms}

For animal experiments, meta-analysis of two studies (Han, 2014; He et al., 2014) showed NGR1 had significant effect on increasing ATP compared with the control group [ $n=28$, SMD: $20.20,95 \%$ 
TABLE 3 | Statement of the characteristics of NGR1.

\begin{tabular}{|c|c|c|c|c|}
\hline Study & Source & Species, concentration & $\begin{array}{l}\text { Quality control reported? } \\
\qquad(\mathrm{Y} / \mathrm{N})\end{array}$ & $\begin{array}{l}\text { Chemical analysis reported? } \\
\text { (Y/N) }\end{array}$ \\
\hline Liu et al., 2010 & $\begin{array}{l}\text { Chinese National Institute for the Control of } \\
\text { Pharmaceutical and Biological Products }\end{array}$ & Panax notoginseng 40 mg/kg & $\mathrm{N}$ & Y-HPLC \\
\hline Deng and Lai 2013 & $\begin{array}{l}\text { Guangxi Wuzhou Pharmaceutical (Group) } \\
\text { Co., Ltd }\end{array}$ & P. notoginseng, $10 \mathrm{mg} / \mathrm{kg}$ & $Y(120502)$ & $\mathrm{N}$ \\
\hline Han, 2014 & $\begin{array}{l}\text { Fengshanjian Medicine Research Co. Ltd. } \\
\text { (Kunming, Yunnan, China) }\end{array}$ & P. notoginseng, $5 \mathrm{mg} / \mathrm{kg}$ & $\mathrm{N}$ & $\mathrm{N}$ \\
\hline He et al., 2014 & Feng-Shan-Jian Medical, Kunming, China & P. notoginseng, 20 mg/kg & $\mathrm{N}$ & $\mathrm{N}$ \\
\hline He et al., 2014 & Feng-Shan-Jian Medical, Kunming, China & P. notoginseng, $0.1 \mathrm{mM}$ & $\mathrm{N}$ & $\mathrm{N}$ \\
\hline Li et al., 2014 & Feng-Shan-Jian Medical (Kunming, China) & P. notoginseng, $10 \mathrm{mg} / \mathrm{kg}$ & $\mathrm{N}$ & $\mathrm{N}$ \\
\hline Meng et al., 2014 & $\begin{array}{l}\text { Shanghai Winherb Medical S \& T } \\
\text { Development (China) }\end{array}$ & P. notoginseng, $20 \mathrm{mg} / \mathrm{kg}$ & $\mathrm{N}$ & Y-HPLC \\
\hline Meng et al., 2014 & $\begin{array}{l}\text { Shanghai Winherb Medical S \& T } \\
\text { Development (China) }\end{array}$ & P. notoginseng, $25 \mu \mathrm{M}$ & $\mathrm{N}$ & Y-HPLC \\
\hline Yu et al., 2014 & Chengdu Must Bio-Technology Co., Ltd & P. notoginseng, $2.5 \mathrm{mg} / \mathrm{kg} / \mathrm{d}$ & Y(MUST-23091001) & Y-HPLC \\
\hline Dong et al., 2015 & $\begin{array}{l}\text { Nanjing ZeLang Medicine Photochemistry } \\
\text { Technology Co., Ltd }\end{array}$ & P. notoginseng, $7 \mathrm{mg} / \mathrm{kg}$ & $\mathrm{N}$ & $\mathrm{N}$ \\
\hline Xia et al., 2015 & $\begin{array}{l}\text { National Institutes for Food andDrug Control } \\
\text { (Beijing, China) }\end{array}$ & P. notoginseng, $60 \mathrm{mg} / \mathrm{kg}$ & N & Y-HPLC \\
\hline Wan et al., 2015 & Chengdu Must Bio-Technology Co., Ltd & P. notoginseng, $100 \mu \mathrm{M}$ & $\mathrm{N}$ & Y-HPLC \\
\hline Wang et al., 2016 & SigmaAldrich & P. notoginseng & $\mathrm{N}$ & N \\
\hline Yu et al., 2016 & $\begin{array}{l}\text { Shanghai Winherb Medical S\&T Development } \\
\text { (Shanghai, China) }\end{array}$ & P. notoginseng, $15 \mathrm{mg} / \mathrm{kg}$ & N & Y-HPLC \\
\hline Yu et al., 2016 & $\begin{array}{l}\text { Shanghai Winherb Medical S\&T Development } \\
\text { (Shanghai, China) }\end{array}$ & P. notoginseng, $20 \mu \mathrm{M}$ & N & Y-HPLC \\
\hline Zhou et al., 2016 & Guangzhou Institute for drug control & P. notoginseng, $10 \mu \mathrm{mol} / \mathrm{L}$ & $\mathrm{N}$ & $\mathrm{N}$ \\
\hline Wang et al., 2016 & SigmaAldrich & P. notoginseng, $20 \mathrm{mmol} / \mathrm{L}$ & $\mathrm{N}$ & Y-HPLC \\
\hline Hou et al., 2017 & Nanjing Jiancheng Bioengineering Institute & P. notoginseng, $20 \mu \mathrm{mol} / \mathrm{L}$ & $\mathrm{N}$ & N \\
\hline Wang et al., 2017 & Sigma-Aldrich & P. notoginseng, $10 \mu \mathrm{mol} / \mathrm{L}$ & $\mathrm{N}$ & Y-HPLC \\
\hline Zhao et al., 2017 & $\begin{array}{l}\text { Shanghai Yuanye Biological Technology Co. } \\
\text { Ltd }\end{array}$ & P. notoginseng, $5 \mathrm{mg} / \mathrm{ml}$ & N & N \\
\hline Zhou et al., 2017 & Guangzhou Institute for drug control & P. notoginseng, $10 \mu \mathrm{M}$ & $\mathrm{N}$ & $\mathrm{N}$ \\
\hline Zou et al., 2017 & $\begin{array}{l}\text { Shanghai Ronghe Pharmaceutical Technology } \\
\text { Development Co., Ltd }\end{array}$ & P. notoginseng, $100 \mathrm{mg} / \mathrm{kg}$ & $\mathrm{N}$ & Y-HPLC \\
\hline Liu et al., 2019 & Sigma-Aldrich & P. notoginseng, $80 \mu \mathrm{M}$ & $\mathrm{N}$ & Y-HPLC \\
\hline Tu et al., 2018 & Sigma-Aldrich & P. notoginseng, $15 \mathrm{mg} / \mathrm{kg}$ & N & Y-HPLC \\
\hline Tu et al., 2018 & Sigma-Aldrich & P. notoginseng, $10 \mu \mathrm{mol} / \mathrm{l}$ & $N$ & Y-HPLC \\
\hline
\end{tabular}

CI: 13.91 to $26.50, P<0.00001$; heterogeneity: $\chi^{2}=0.16, \mathrm{df}=1$ $\left.(P=0.69), \mathrm{I}^{2}=0 \%\right]$ (Figure 9). One study (He et al., 2014) showed that NGR1 increased the proportion of anti-apoptosis proteins such as Bcl-2/Bax and cleaved caspase-3/procaspase-3 compared with the control group $(P<0.05)$; one study (Yu et al., 2014) showed that NGR1 increased the density of neovasculature and improved the expression of angiogenesis protein such as VEGF and bFGF compared with the control group $(P<0.05)$; one study (He et al., 2014) showed that NGR1 improved the production of ATP and ATP5D compared with the control group $(P<0.05)$; one study (He et al., 2014) demonstrated that NGR1 reduced the protein level of phosphorylation-AMP-activated protein kinase (P-AMPK), Rho-associated coil kinase (ROCK), and phosphorylation-myosin phosphatase target subunit-1 (P-MYPT1) compared with the control group $(P<0.05)$; one study (Xia et al., 2015) indicated that NGR1 decreased the inflammatory mediators such as IL- $1 \beta$ and IL- 8 compared with the control group $(P<0.05)$; one study (Xia et al., 2015) indicated that NGR1 decreased the expression of phosphorylation-nuclear factor-кBp65 (p-NF-кBp65) and phosphorylation-nuclear factor of kappa light polypeptide gene enhancer in B-cells inhibitor alpha ( $\mathrm{p}-\mathrm{I} \kappa \mathrm{B} \alpha)$ compared with the control group $(\mathrm{P}<0.05)$; one study (Yu et al., 2016) indicated that NGR1 lowered the level of ERS-responsive proteins, such as glucose regulated protein 78 (GRP78), phospho-protein kinase R-like ER kinase (P-PERK), activating transcription factor 6 (ATF6), and inositol-requiring enzyme-1a (IRE1) compared with the control group $(P<0.05)$; one study (Meng et al., 2014) indicated that NGR1 decreased the expression of 8-hydroxy-2 deoxyguanosine (8-OHDG) compared with the control group $(P<0.05)$; one study (Tu et al., 2018) demonstrated that NGR1 increased the synthesis of ribosomal translation regulatory proteins such as phosphomammalian target of rapamycin (P-mTOR), phospho-protein S6 kinase (P-P70S6K), and phospho-eukaryotic initiation factor $4 \mathrm{E}$ binding protein 1 (P-4EBP-1) compared with the control group $(P<0.05)$; one study (Tu et al., 2018) demonstrated that NGR1 reduced the expression of pro-apoptotic proteins, such as phospho-c-Jun N-terminal kinase (P-JNK) compared with the control group $(P<0.05)$; one study (Zhou et al., 2016) showed that NGR1 decreased the level of extracellular regulated protein kinases $(\mathrm{ERK} 1 / 2)$ and $\mathrm{p} 38$-mitogen-activated protein kinase (p38MAPK) compared with the control group $(P<0.01)$. 
TABLE 4 | Risk of bias of the included in vivo studies.

\begin{tabular}{|c|c|c|c|c|c|c|c|c|c|c|c|}
\hline Study & A & B & C & D & $\mathbf{E}$ & $\mathbf{F}$ & G & $\mathbf{H}$ & $\mathbf{I}$ & $\mathbf{J}$ & Total \\
\hline $\begin{array}{l}\text { Deng and Lai } \\
2013\end{array}$ & $\sqrt{ }$ & & $\sqrt{ }$ & & & $\sqrt{ }$ & & & & & 3 \\
\hline Han, 2014 & $\sqrt{ }$ & $\sqrt{ }$ & $\sqrt{ }$ & & & $\sqrt{ }$ & & & & & 4 \\
\hline He et al., 2014 & $\sqrt{ }$ & $\sqrt{ }$ & $\sqrt{ }$ & $\sqrt{ }$ & & $\sqrt{ }$ & & & $\sqrt{ }$ & $\sqrt{ }$ & 7 \\
\hline Yu et al., 2014 & $\sqrt{ }$ & & $\sqrt{ }$ & $\sqrt{ }$ & & $\sqrt{ }$ & & & & $\sqrt{ }$ & 5 \\
\hline Xia et al., 2015 & $\sqrt{ }$ & $\sqrt{ }$ & $\sqrt{ }$ & $\sqrt{ }$ & & $\sqrt{ }$ & & & $\sqrt{ }$ & $\sqrt{ }$ & 7 \\
\hline Yu et al., 2016 & $\sqrt{ }$ & $\sqrt{ }$ & $\sqrt{ }$ & $\sqrt{ }$ & $\sqrt{ }$ & $\sqrt{ }$ & & & $\sqrt{ }$ & $\sqrt{ }$ & 8 \\
\hline Tu et al., 2018 & $\sqrt{ }$ & $\sqrt{ }$ & & & & $\sqrt{ }$ & & & $\sqrt{ }$ & $\sqrt{ }$ & 5 \\
\hline Meng et al., 2014 & $\sqrt{ }$ & $\sqrt{ }$ & $\sqrt{ }$ & & $\sqrt{ }$ & $\sqrt{ }$ & & & $\sqrt{ }$ & $\sqrt{ }$ & 7 \\
\hline Zou et al., 2017 & $\sqrt{ }$ & $\sqrt{ }$ & $\sqrt{ }$ & & & $\sqrt{ }$ & & & $\sqrt{ }$ & & 5 \\
\hline Wang et al., 2016 & $\sqrt{ }$ & $\sqrt{ }$ & & & & $\sqrt{ }$ & & & $\sqrt{ }$ & $\sqrt{ }$ & 5 \\
\hline Zhao et al., 2017 & $\sqrt{ }$ & $\sqrt{ }$ & & & & $\sqrt{ }$ & & & & & 3 \\
\hline Dong et al., 2015 & $\sqrt{ }$ & $\sqrt{ }$ & & & & $\sqrt{ }$ & & & & $\sqrt{ }$ & 4 \\
\hline
\end{tabular}

Studies fulfilling the criteria of: $A$, peer reviewed publication; $B$, control of temperature; $C$, random allocation to treatment or control; $D$, blinded induction of model; E, blinded assessment of outcome; F, use of anesthetic without significant intrinsic vascular protection activity; G, appropriate animal model (aged, diabetic, or hypertensive); H, sample size calculation; I, compliance with animal welfare regulations; J, statement of potential conflict of interests.

TABLE $\mathbf{5}$ | Risk of bias of the included in vitro studies.

\begin{tabular}{|c|c|c|c|c|c|c|c|c|c|c|c|}
\hline Study & A & B & C & D & $\mathbf{E}$ & $\mathbf{F}$ & G & $\mathbf{H}$ & 1 & $J$ & Total \\
\hline Wan et al., 2015 & $\sqrt{ }$ & & & & $\sqrt{ }$ & & & & & $\sqrt{ }$ & 3 \\
\hline Liu, 2018 & $\sqrt{ }$ & $\sqrt{ }$ & & $\sqrt{ }$ & $\sqrt{ }$ & & & & & $\sqrt{ }$ & 5 \\
\hline Meng et al., 2014 & $\sqrt{ }$ & $\sqrt{ }$ & & $\sqrt{ }$ & $\sqrt{ }$ & & & & & $\sqrt{ }$ & 5 \\
\hline Wang, 2016 & $\sqrt{ }$ & $\sqrt{ }$ & & $\sqrt{ }$ & $\sqrt{ }$ & & & & & $\sqrt{ }$ & 5 \\
\hline Tu et al., 2018 & $\sqrt{ }$ & $\sqrt{ }$ & & $\sqrt{ }$ & $\sqrt{ }$ & & & & & $\sqrt{ }$ & 5 \\
\hline
\end{tabular}

Studies fulfilling the criteria of: A, peer-reviewed publication; B, use of appropriate primary cells to study; $C$, cell lines with reliable source or validated by appropriate methods; , D, assess toxicity of treatment on cells; $E$, culture environment (culture media/sera, $\mathrm{pH} / \mathrm{CO}_{2}$ and temperature); $F$, random allocation to treatment or control; $\mathrm{G}$, blinded induction of model; $\mathrm{H}$, blinded assessment of outcome; I, calculation of the sample size necessary to achieve sufficient power; and $\mathrm{J}$, statement of potential conflict of interests. Each item was awarded one point.

We summarized a schematic representation for the possible intrinsic mechanisms of NGR1 protection for organ I/R injury (Figure 10).

\section{DISCUSSION}

\section{Summary of Evidence}

To our knowledge, this is a first SR to assess the preclinical evidences of NGR1 for I/R injury both in vivo and in vitro. Twenty-five studies with 304 animals and 124 cells were selected. The quality of the included studies was generally moderate. In the present study, NGR1 exerts multiple organ protection in I/R injury, mainly through antioxidant, anti-apoptosis, and anti-inflammatory, promoting angiogenesis and improving energy metabolism.

\section{Limitations}

None of these studies had used animals with comorbidities such as diabetes, hypertension, or hyperlipidemia. Primary cells were considered to be the suitable subjects to validate organ protective function in vitro, while only two studies (Xia et al., 2015; Yu et al., 2016) used subcultured cells (H9C2). None of these studies reported the blindness of ischemia induction, randomized allocation to treatment, or control group and sample size calculation. In most studies, the signal pathways investigated could not fully validate the therapeutic targets.

\section{Implications}

Our findings demonstrated that NGR1 could attenuate I/Rinduced organ injuries by a variety of pathways. For myocardial I/R injury, NGR1 could reduce MI size, increase cardiomyocyte viability, and exert cardioprotective function by the following mechanisms: 1) anti-inflammatory effect by activating the VDUP1/NF- $\kappa B$ signaling pathway (Xia et al., 2015) and decreasing the expression of ICAM-1 and CD18 (Han, 2014); 2) improving energy metabolism via up-regulation of ROCK signaling pathway (He et al., 2014); 3) promoting angiogenesis by increasing the expression of VEGF and bFGF (Yu et al., 2014); 4) inhibiting apoptosis by decreasing the expression level of ERS-responsive proteins such as GRP78, P-PERK, ATF6, 


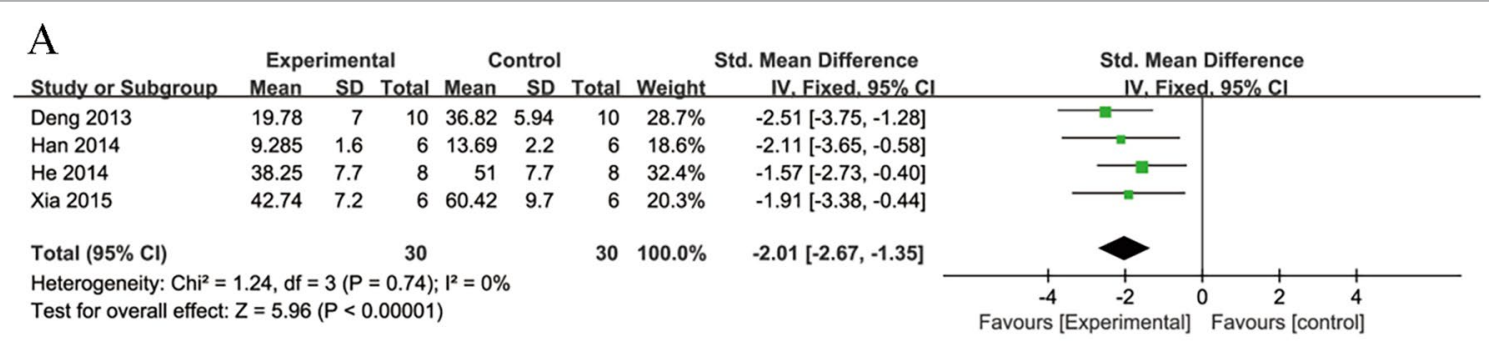

B

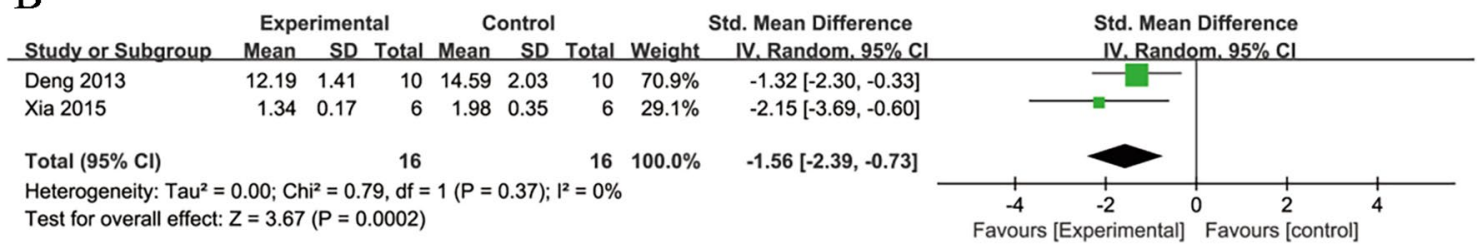

$\mathrm{C}$

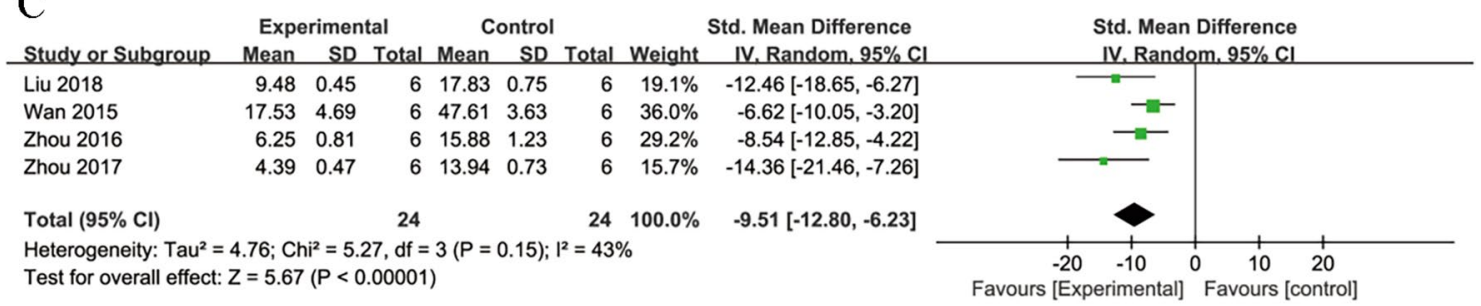

FIGURE 3 | The forest plot: (A) effects of notoginsenoside R1 for reducing the myocardial infarction size compared with the control group ( $\mathrm{n}=30$ per group). (B) Effects of notoginsenoside R1 for reducing the creatine kinase compared with the control group ( $n=16$ per group). (C) The forest plot: effects of notoginsenoside R1 for reducing cardiomyocytes apoptosis rate compared with the control group ( $n=24$ per group).

\begin{tabular}{|c|c|c|c|c|c|c|c|c|c|}
\hline \multirow[b]{2}{*}{ Study or Subgroup } & \multicolumn{3}{|c|}{ Experimental } & \multicolumn{2}{|c|}{ Control } & & \multirow{2}{*}{$\begin{array}{l}\text { Std. Mean Difference } \\
\text { IV, Random, } 95 \% \mathrm{Cl}\end{array}$} & \multirow{2}{*}{\multicolumn{2}{|c|}{$\begin{array}{r}\text { Std. Mean Difference } \\
\text { IV. Random, } 95 \% \mathrm{Cl}\end{array}$}} \\
\hline & Mean & SD & Total $n$ & Mean & SD & Total Weight & & & \\
\hline He 2014 & 84.06 & 2.9 & 65 & 55.07 & 2.2 & $614.6 \%$ & $10.40[5.20,15.60]$ & & - \\
\hline Liu 2018 & 77.84 & 2.84 & 64 & 46.02 & 3.98 & $20.4 \%$ & $8.50[4.20,12.79]$ & & $-\square$ \\
\hline Wan 2015 & 88 & 2 & 6 & 59 & 3 & $14.3 \%$ & $10.50[5.25,15.75]$ & & - \\
\hline Yu 2016 & 90 & 3.07 & 65 & 55.39 & 0.77 & $8.3 \%$ & $14.27[7.22,21.33]$ & & \\
\hline Zhou 2016 & 95 & 7 & 6 & 58 & 2.5 & $30.4 \%$ & $6.50[3.13,9.87]$ & & - \\
\hline Zhou 2017 & 89 & 3.3 & 6 & 56 & 1.7 & $12.1 \%$ & $11.60[5.83,17.38]$ & & \\
\hline Total $(95 \% \mathrm{Cl})$ & & & 36 & & & $36 \quad 100.0 \%$ & $9.31[7.21,11.41]$ & & 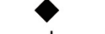 \\
\hline $\begin{array}{l}\text { Heterogeneity: } \text { Tau }^{2}= \\
\text { Test for overall effect: }\end{array}$ & $\begin{array}{l}0.82 ; C h \\
Z=8.69\end{array}$ & $\begin{array}{l}i^{2}=5.6 \\
(P<0 .\end{array}$ & $\begin{array}{l}3, d f=5 \\
00001)\end{array}$ & $5(P=0$ & $0.34) ; l^{2}$ & $2=12 \%$ & & $\begin{array}{ccc}-20 & -10 & 0 \\
\text { Favours [control] }\end{array}$ & $\begin{array}{c}10 \\
\text { Favours }[\mathrm{E}\end{array}$ \\
\hline
\end{tabular}

FIGURE 4 | The forest plot: effects of notoginsenoside R1 for increasing cardiomyocytes cell viability compared with the control group ( $n=36$ per group).

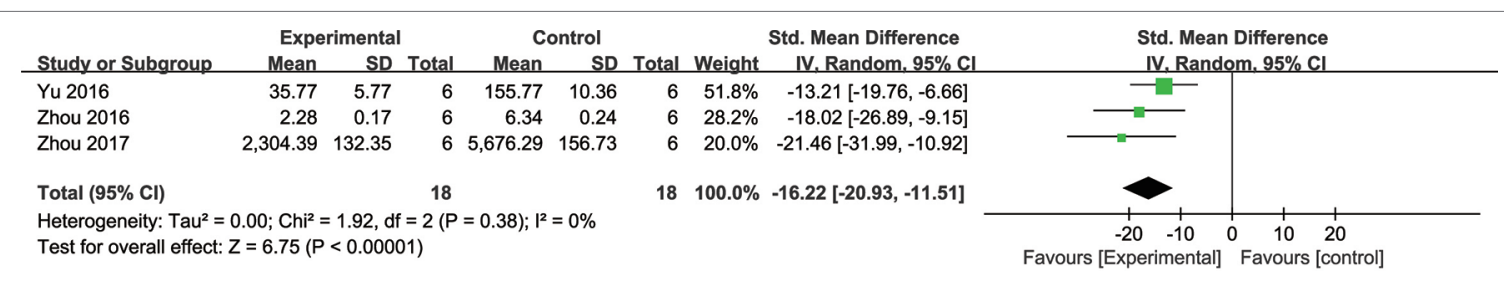

FIGURE 5 | The forest plot: effects of notoginsenoside R1 for reducing cardiomyocytes LDH release compared with the control group ( $\mathrm{n}=18$ per group).

and IRE, and reducing the expression of pro-apoptosis proteins such as CHOP, Caspase-12, and P-JNK (Yu et al., 2016); and 5) improving microvascular dysfunction by increasing the expression of tight junction proteins such as ZO-1, VE, JAM-1, and Claudin-5 and reducing the level of embrane intrinsic protein of Cav-1 and Cav-3 (Han, 2014). For cerebral I/R injury, NGR1 could decrease cerebral infarction volume (Meng et al., 2014; Dong et al., 2015; Wang et al., 2016; Zou et al., 2017; Tu et al., 2018) and neurological deficit score (Meng et al., 2014; Dong et al., 2015) and increase cerebral cell viability 


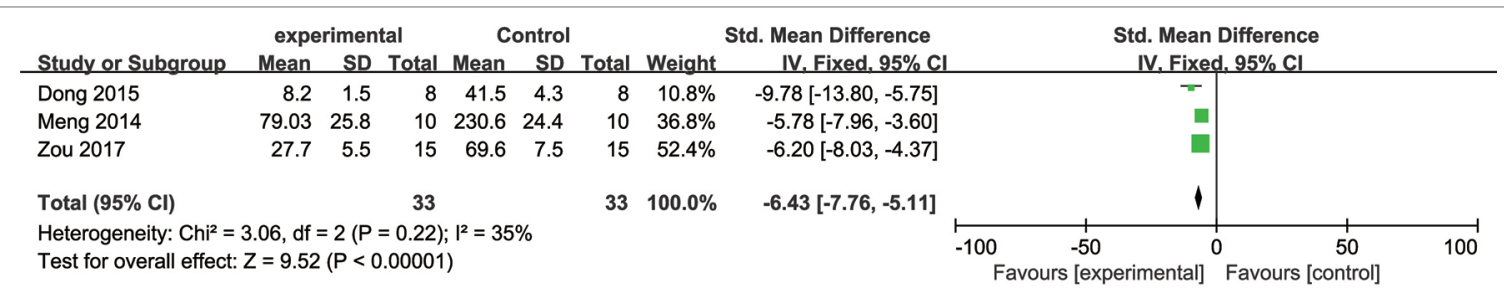

FIGURE 6 | The forest plot: effects of notoginsenoside R1 for reducing cerebral infarction volume compared with the control group ( $n=33$ per group).

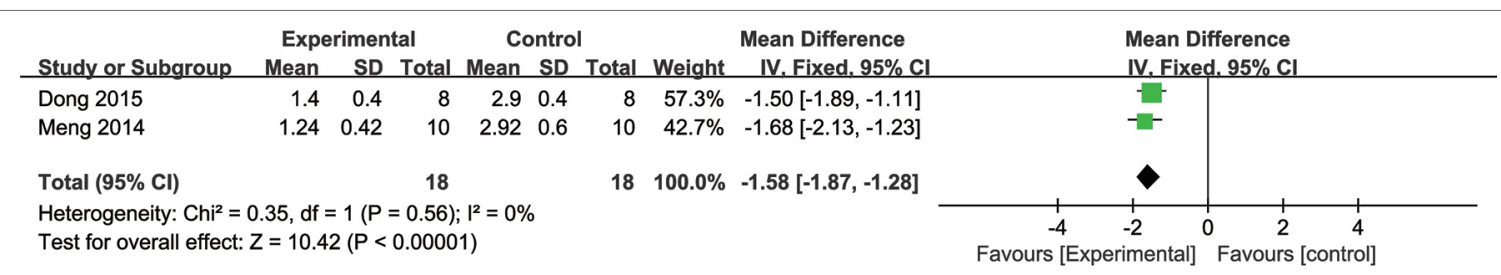

FIGURE 7 | The forest plot: effects of notoginsenoside R1 for reducing neurologic deficit score compared with the control group ( $\mathrm{n}=18$ per group).

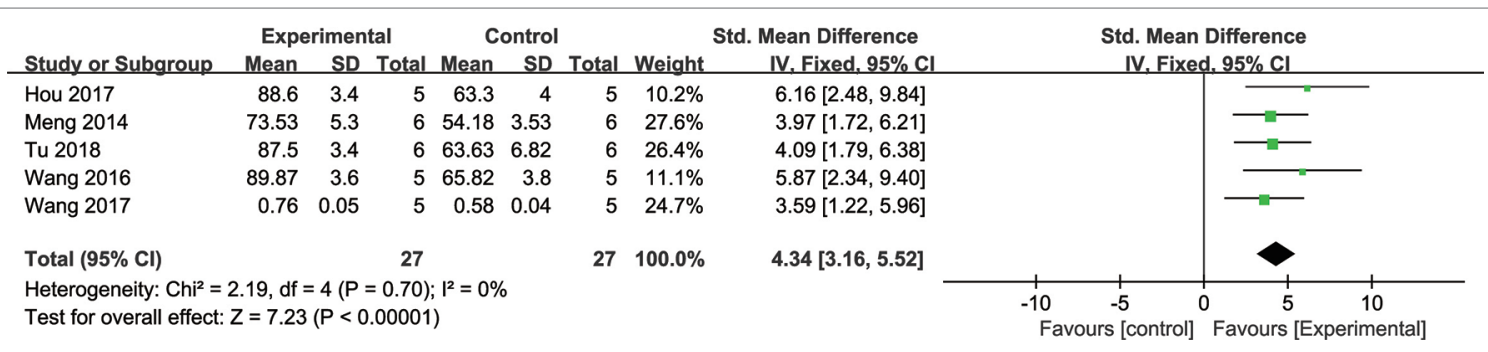

FIGURE 8 | The forest plot: effects of notoginsenoside R1 for increasing cerebral cell viability compared with the control group $(n=27$ per group).

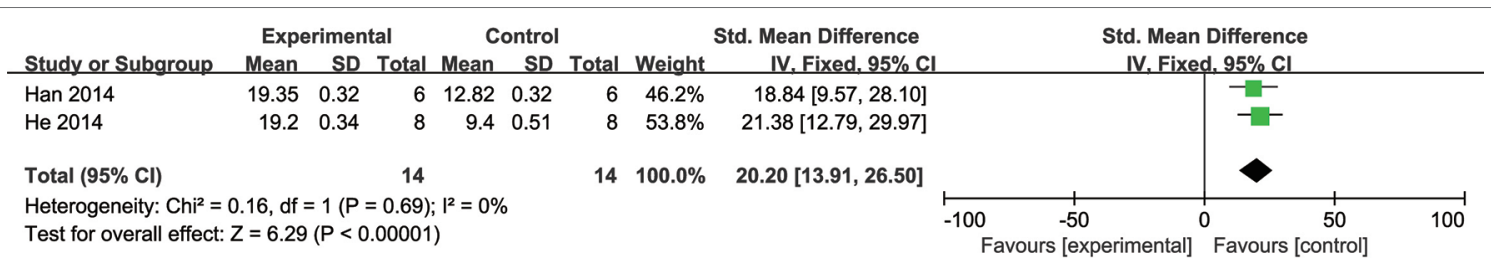

FIGURE 9 | The forest plot: effects of notoginsenoside R1 for increasing ATP compared with the control group ( $n=14$ per group).

(Wang et al., 2016; Hou et al., 2017; Wang et al., 2017; Tu et al., 2018). The neuroprotective effect of NGR1 was mainly due to anti-apoptosis effect by regulating the Akt/Nrf2 and Akt/ mTOR/JNK pathways (Tu et al., 2018). For renal I/R injury, NGR1 had an anti-inflammatory effect through the p38MAPK/ NFkB pathway and also increased the proportion of bcl-2/bax, which could produce anti-apoptosis effect (Liu et al., 2010). For intestinal I/R injury, NGR1 could attenuate the production of inflammatory cytokine by inhibiting the NF- $\kappa B$ pathway and reduce the expression of loss of tight junction proteins such as zonula occluden-1 (ZO-1), occludin, and claudin-5. It was also reported that NGR1 could improve the energy metabolism via depressing ATP5D expression during intestinal I/R injury ( $\mathrm{Li}$ et al., 2014). Although NGR1 could exert organ-protective effect in I/R injury via multiple signal pathways, the validation of NGR1 therapeutic target is insufficient. In the process of drug discovery, it is common to perform target validation to illustrate 1) the function of the potential target in disease phenotype 2) and the therapeutic efficacy of drug-like molecules through modulating the activities of the potential target (Zhu et al., 2012; Gashaw et al., 2012). The approach of validation generally included gene knockout/knockdown in vivo, pharmacological inhibitors, and small interfering RNA (SiRNA) of cells in vitro (Overall and Kleifeld 2006). In the present study, four studies (He et al., 2014; Meng et al., 2014; Yu et al., 2016; Tu et al., 2018) used signal pathway inhibitors to validate the drug targets. However, the remaining studies were not verified. Although inhibitors enjoy strong suppression functions and high selectivity, the severe side effects in organisms mean that this is not the best way to study potential targets for drugs (May et al., 2011; Yan 2016). 
NGR1

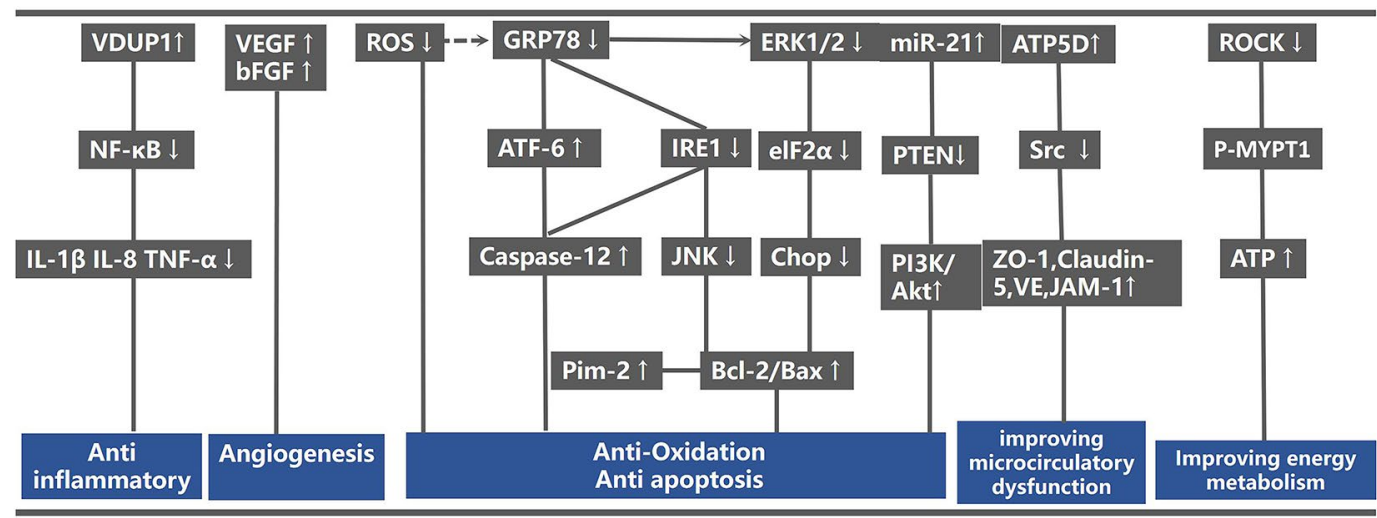

Cardioprotection

NGR1

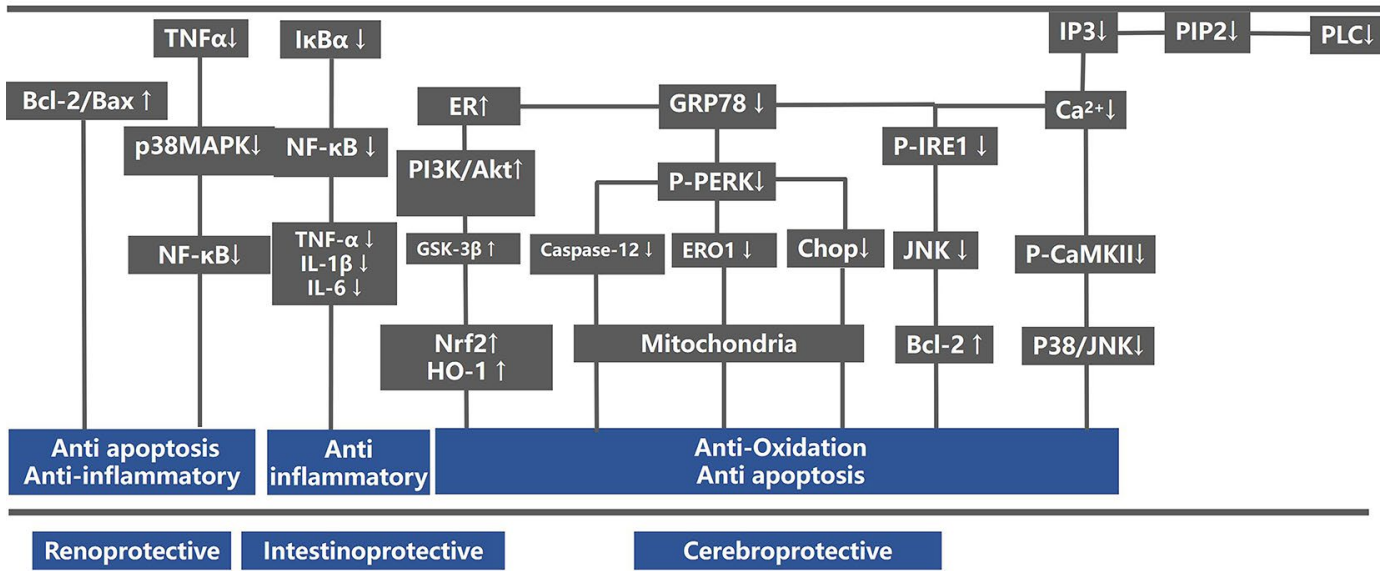

FIGURE 10 | A schematic representation of cardioprotective mechanisms of NGR1 for organs ischemia/reperfusion injury. VDUP1, vitamin D3 up-regulated protein 1; NF-kB, nuclear factor-kappa; IL-1, interleukin-1; IL-1 $\beta$, interleukin 1 beta; IL-8, interleukin-8; TNF- $\alpha$, tumor necrosis factor- $\alpha$; VEGF, vascular endothelial growth factor; bFGF, basic fibroblast growth factor; GRP78, glucose regulated protein 78; PERK, protein kinase R-like ER kinase; ATF-6, activating transcription factor 6; IRE1, inositol-requiring enzyme- $1 \alpha$; JNK, Jun N-terminal kinase; CHOP, C/EBP homologous protein; B-cell lymphoma-2; Bax, BCL2-associated X protein; elf2 $\alpha$, eukaryotic initiation factor $2 \alpha$; PTEN, phosphatase and tensin homolog deleted on chromosome ten; PI3K, p-mTOR, phospho-mammalian target of rapamycin; ZO-1, Zonula occludens-1; JAM-1, recombinant junctional adhesion molecule 1; Cav-1, caveolin 1; Cav-3, caveolin 3; MYPT1, myosin phosphatase target subunit-1; ATP, adenosine triphosphate; ROCK, Rho-associated kinase; GSK-3 $\beta$, glycogen synthase kinase-3 beta; IкB $\alpha$, nuclear factor of kappa light polypeptide gene enhancer in B-cells inhibitor; alpha; HO-1, heme oxygenase-1; Nrf2, nuclear factor erythroid 2-related factor 2; IP3, inositol 1, 4, 5-trisphosphate; PLC, phospholipase C.

Owing to different isoforms may hold different functions, gene knockout technique effectively eliminates all isoforms possessing alternative splicing of the precursor messenger RNAs (mRNAs) and post-translational modifications compared with RNA interference (Smith, 2003). Unfortunately, it is widespread that off-target effects highly occurred in gene knockdown (Kok et al., 2015). Thus, a call for researchers should utilize animal with gene knockout to validate the drug target.

A multitude of preclinical researches showed that many available medical treatment strategies share good efficacy on animal models, while they have poor efficacy in clinical practice (Worp and Sandercock, 2012). High-quality preclinical study can provide vital information for justifying clinical advancement (Henderson et al., 2013). An SR of preclinical research is a novel method that offers important information for future clinical trials. According to CAMARADES 10-item checklist, the score of the included studies was medium. They are similar to other animal studies, and the main limitations were that no study reported sample size calculation, allocation concealment, and blinding of outcome assessment (Bao et al., 2018). Inadequate 
sample size could not reach the approximately threshold with sufficient power and efficacy (Bacchetti 2010). Blinding of the ischemia model refers to establishing ischemia models for experimental animals firstly and then assigning randomly. This method can avoid selection bias in allocating the animals to the treatment groups and makes it more likely that the intergroups are comparable (Festing and Altman, 2002). An overview study involving seven meta-analyses demonstrated that unblinded induction of ischemia had a greater effect size (about 13.1\%) than studies that included blinding (Crossley et al., 2008). Thus, the poor blinding of the ischemia model induction and outcome evaluation may lead to overestimating efficacy in preclinical studies (Crossley et al., 2008). Those who were attacked with IHD tend to possess an advanced age and coexist with diabetes mellitus, hyperglycemia, and hypertension (Heusch 2017; Davidson et al., 2019). However, investigators prefer to utilize young healthy animals to conduct research. This may overestimate the efficacy of intervention compared with clinical administration (Sanne et al., 2014). Accordingly, researchers should take the following factors into account: 1) improving the quality of study design; 2) blinding for induction of ischemia models and evaluation of outcomes; 3) reporting completely experimental program (sample size calculation, allocation concealment) and result; and 4) utilizing animals with comorbidities that can maximally mimic the ischemia patients suffering from hypertension, hyperlipidemia, and diabetes.

The animal model that holds maximum replication of important functional, structural, and molecular pathological characteristics of human disease is crucial to clinical translation (Saulnier-Blache et al., 2018). LAD ligation in rats is the most common I/R injury model (Nishina et al., 2001; Macarthur et al., 2013). MCA occlusion is a widely accepted model to mimic human stroke and to explore the mechanism of stroke (Durukan and Tatlisumak 2007). In the present study, most of the included studies selected these two recognized models, except one study (Deng and Lai, 2013) by injecting pituitrin and three studies (Wang et al., 2016; Tu et al., 2018; Zou et al., 2017) via ligating one or bilateral common carotid artery. Small animals such as mice and rats are the most commonly used vertebrate species because of their small size, low cost, easy handling, and fast reproduction rate (Seabrook et al., 2017). However, compared with humans, small animals have some limitations with small body size, short lifetime, and fundamentally distinct physiology (Cibelli et al., 2013). Large animal models are highly similar with human anatomy and pharmacodynamics, which can provide a choice of appropriate animal models for particular human disease conditions and medical applications (Van Hout et al., 2015). However, it also has weaknesses such as more expensive, difficult to manipulate, and ethical issues. Thus, we should select an ideal model that usually is a biological representative of human disease, inexpensive, reproducible, easily manipulated, and ethically sound according to experimental purpose.

A powerful outcome measure is essential to certify the efficacy of new therapy (Hietamies et al., 2018). In MI research, LVEF is the strongest predictor factor for post-MI both clinically (Barthel et al., 2013) and preclinically (Kanelidis et al., 2017). Cardiac troponins, especially the cTnI and cTnT, are the preferred biomarkers for the diagnosis and prognosis of MI clinically (Thygesen et al., 2012; Hall et al., 2015). Plasma cTnI can also serve as a predictor for myocardial injury in small animal models (Frobert et al., 2015). In cerebral ischemia, perfusionweighted magnetic resonance imaging is an identified predictor, which can evaluate the ischemic stroke patients with perfusion abnormalities (Sasaki et al., 2011; Powers et al., 2018). In renal ischemia, GFR is an essential indicator to predict prognosis for renal injury, which can reflect the efficacy of intervention through renal blood flow and regional perfusion in the clinical setting (Dagher et al., 2003). However, few present included studies selected these important outcomes. Thus, they should be selected in priority of further myocardial I/R injury studies.

Primary cell with wild-type and unadulterated nature more closely mimics the physiological state of cells in vivo and generates more relevant data representing living systems (Astashkina et al., 2012; Egger et al., 2013). In nerve cell I/R models, all cells were harvested from the cerebral cortices of rat fetuses. However, in cardiomyocytes I/R models, two studies (He et al., 2014; Wan et al., 2015) used cell lines (H9C2), which did not accurately simulate the physiological and pathological state of the cells in vivo. Cell viability examination is a routine assay that can quantitatively assess cell reaction to drug administration. Meanwhile, the optimal drug concentration is vital for pharmacological research in cellular models, which can achieve maximum efficacy while ensuring drug safety, providing a reference for further in vivo experiments (Astashkina et al., 2012; Bao et al., 2018). 3-(4,5-Dimethyl-2-thiazolyl)-2,5-diphenyl-2Htetrazolium bromide (MTT) and Cell Counting Kit-8 (CCK-8) are two classical assay methods used to detect cell viability and sift the optimal drug concentration. Eight studies (Zhou et al., 2016; Wan et al., 2015; Yu et al., 2016; Wang et al., 2016; Hou et al., 2017; Wang et al., 2017; Zhou et al., 2017; Tu et al., 2018) used MTT assay, and two studies (He et al., 2014; Meng et al., 2014; Liu et al., 2019) used the CCK- 8 assay. However, one study (He et al., 2014) did not conduct the assay of screening the optimal drug concentration. OGD/R is a good method for mimicking the general pathophysiological process of I/R injury at the cellular level (Jones et al., 2004). However, there are various methods for constructing cell hypoxia. Many homemade devices or chemical agents are used to create hypoxia environment. The oxygen concentration is not uniform, and the time of hypoxia varies greatly, which makes the research results to lack comparability. In addition, all included studies did not report allocation concealment, blinding of model induction, and blinding of outcome assessment. Thus, we recommend that researchers should utilize primary cells and the appropriate methods of induction in study, select optimal drug concentration, and report experimental protocols with complete information. Standardized preclinical research reporting, suitable animal/cell models, and appropriate primary outcome measures are crucial to translation from bench to bed. In order to better explore the protective function of NGR1 in I/R injury of multiple organs, researchers should add more animal/cell experiments in other less studied organs such as the liver, lung, renal, and intestinal ischemia. 
To date, there has been no available therapy clinically that can alleviate I/R injury (Cung et al., 2015). An enduring ischemia leads to irreversible damage for cardiomyocytes and neurons with the low ability to regenerate or renew (Galluzzi et al., 2009; Jennings 2013). And delayed reperfusion is associated with high mortality in patients after primary PCI (Terkelsen et al., 2010). In the present study, seven studies (Deng and Lai, 2013; Li et al., 2014; Li et al., 2014; Han, 2014; He et al., 2014; Meng et al., 2014; Dong et al., 2015; Xia et al., 2015) had reported that experimental subjects were pretreated with NGR1 before modeling, and the rest of the studies had not exploited pretreatment methods. Owing to the slow onset time of herbal efficacy, administration of herbs before the induction of models was often used for herbal pharmacological researches in order to reach the effective plasma concentration. Thus, pretreatment of NGR1 before molding establishment exerts a therapeutical role rather than an approach of preventive treatment. In addition, the treatment at post-model induction is more in line with clinical practice. Definitely, it is more appropriate to study herbal pharmacology in both two different administrations.

NGR1 belongs to 20(S)-protopanaxatriol type ginsenoside, which possesses glycosides moieties at C-6 and/or C-20. NGR1 has a poor bioavailability due to fast elimination from plasma and low permeability (Zhang et al., 2019). And the direct absorption of natural ginsenosides is quite not easy due to the fact that they need to be transformed into secondary saponins via the metabolism of gut, and then they can be easily absorbed and utilized in the blood (Liu et al., 2009). Previous study showed a low permeability of the main metabolites of NGR1 across the Caco- 2 cell monolayer, which implies a poor absorption $(<1 \%)$ in humans after being taken orally; however, the permeability of the metabolites of NGR1 in intestinal bacteria is higher than that in the Caco- 2 cell monolayer (Ruan et al., 2010). These findings may suggest that metabolites of NGR1 forming by intestinal bacteria have a better effect than NGR1. They are eliminated from the gastrointestinal tract to produce a series of disaccharide glycosides, including ginsenoside $\operatorname{Rg} 1$, notoginseng saponins $\mathrm{R} 2$, ginseng saponins Rh1, ginsenosides $\mathrm{F} 1$, and glycoside ligand proglycerol (Liu et al., 2009). The four metabolites of NGR1, including ginsenoside $\mathrm{Rg} 1$, notoginsenoside R2, ginsenoside Rh1, ginsenoside F1, and the aglycone protopanaxatriol, have been identified and display relative high exploration in rat plasma (Zhang et al., 2019). In vitro experiments mainly include the following functions: 1) carrying on experiment in Caco-2 cell to evaluate the absorption of compounds through the inner layer of the gastrointestinal tract (Artursson et al., 2001);2) identifying the disposition of compounds among organs to study the distribution mechanism; and 3) studying and quantitating the metabolism of chemicals (Pelkonen and Turpeinen 2007). The role of experimental studies in vitro of the review mainly sifts the optimum concentration and explores the signaling pathway, while experimental studies in vivo mainly focus on the change of infarct size and the indicator relating to clinical trials (such as creatine kinase, neurologic deficit score). Previous studies have shown that the results of intervention in vitro extrapolating to the observation in vivo remains very challenging. (Wienkers and Heath, 2005; Joris et al., 2013; Hadjidemetriou et al., 2015). In the present review, the included studies did not test the metabolites of NGR1 to explore the protection mechanism on ischemic diseases. Recent studies have shown that ginsenoside metabolites share better biological effects than those of ginsenosides (Feng et al., 2017). Studies have shown that the metabolites of ginsenosides $\mathrm{Rb} 1$ can form rare ginsenosides such as Rg3, Rd, F2, and compound $\mathrm{K}$ with high bioactive functions by physical and biological treatments (Oh and Kim 2016; Fu et al., 2017). Ginsenosides Rg3 has a strong protection on cerebral ischemia compared with other ginsenosides such as Rg1, Rh2, and Rg5 (Cheng et al., 2019). Compound K showed great protection on Alzheimer's disease and cerebral ischemia via enhancing cognition effects and decreasing inflammatory biomarkers (Oh and Kim 2016), and has protective effects on myocardial IR injury via reducing infarct size and activating the PI3K pathway (Tsutsumi et al., 2011). Similarly, ginsenoside and notoginsenoside belong to same group of triterpenoid saponins, and thus we extrapolated that NGR1 should own the same property: the metabolites of NGR1 have a greater efficacy than NGR1. Owing to the lack of direct evidence of the metabolites of NGR1, they should be further tested directly in order to extend the findings of animal models to the clinic. Currently, the application of metabolomics in herbal medicine is a research focus (Wang et al., 2017; Bi et al., 2017). So, further study should test the metabolites of NGR1 directly and one of the proper methods through using metabolomics.

\section{CONCLUSION}

The findings of the present study demonstrated that NGR1 exerts organ protective functions for I/R injury, mainly through antioxidant, anti-inflammatory, and anti-apoptosis, increasing energy metabolism and angiogenesis. Further translation studies are needed.

\section{DATA AVAILABILITY STATEMENT}

The datasets generated for this study are available on request to the corresponding author.

\section{AUTHOR CONTRIBUTIONS}

QT, P-CZ, ZZ, L-HD, Z-HW, HZ, G-QZ, and YW designed the study. ZZ, L-HD, and Z-HW collected the data. QT, P-CZ, $\mathrm{HZ}$, and ZZ performed all analyses. QT and P-CZ wrote the manuscript.

\section{FUNDING}

This work was supported by the grant of the National Natural Science Foundation of China (81573750/81473491/81173395/ H2902). 


\section{REFERENCES}

Alex, D., Felipe, D. P., Giovanni, S., Lach, A. B., Galfo, F., Mendonca, B. P., et al. (2017). Ammonium tetrathiomolybdate following ischemia/reperfusion injury: Chemistry, pharmacology, and impact of a new class of sulfide donor in preclinical injury models. PLOS Med. 14 (7), e1002310-. doi: 10.1371/journal. pmed.1002310

Artursson, P., Palm, K., and Luthman, K. (2001). Caco-2 monolayers in experimental and theoretical predictions of drug transport. Adv. Drug Deliv. Rev. 46. doi: 10.1016/S0169-409X(00)00128-9

Astashkina, A., Mann, B., and Grainger, D. W. (2012). A critical evaluation of in vitro cell culture models for high-throughput drug screening and toxicity. Pharmacol. Ther. 134 (1), 82-106. doi: 10.1016/j.pharmthera.2012.01.001

Bacchetti, P. (2010). Current sample size conventions: flaws, harms, and alternatives. BMC Med. 8 (1), 17-10. doi: 10.1186/1741-7015-8-17 8,1(2010-03-22).

Bao, X. Y., Zheng, Q., Tong, Q., Zhu, P. C., Zhuang, Z., Zheng, G. Q., et al. (2018). Danshensu for Myocardial Ischemic Injury: Preclinical Evidence and Novel Methodology of Quality Assessment Tool. Front. Pharmacol. 9, 1445. doi: 10.3389/fphar.2018.01445

Barthel, P., Wensel, R., Bauer, A., Muller, A., Wolf, P., Ulm, K., et al.. (2013). Respiratory rate predicts outcome after acute myocardial infarction: a prospective cohort study. Eur. Heart J. 34 (22), 1644-1650. doi: 10.1093/ eurheartj/ehs 420

Bi, W., Liu, H., Shen, J., Zhang, L. H., Li, P., Peng, B., et al. (2017). Chemopreventive effects of ku-jin tea against aom-induced precancerous colorectal lesions in rats and metabolomic analysis. Sci. Rep. 7 (1), 15893. doi: 10.1038/ s41598-017-16237-0

Chen, G. Y., and Nuñez, G. (2010). Sterile inflammation: sensing and reacting to damage. Nat. Rev. Immunol. 10 (12), 826-837. doi: 10.1038/nri2873

Cheng, Z. K., Zhang, M., Ling, C. L., Zhu, Y., Ren, H. W., Hong, C., et al. (2019). Neuroprotective Effects of Ginsenosides against Cerebral Ischemia. Molecules 2019, 24(6). doi: 10.3390/molecules24061102

Cibelli, J., Emborg, M. E., Prockop, D. J., Roberts, M., Schatten, G., Rao, M., et al. (2013). Strategies for improving animal models for regenerative medicine. Cell Stem Cell 12 (3), 271-274. doi: 10.1016/j.stem.2013.01.004

Crossley, N. A., Sena, E., Goehler, J., Horn, J., Bart, V. D. W., Bath, P. M. W., et al. (2008). Empirical evidence of bias in the design of experimental stroke studies: a metaepidemiologic approach. Stroke 39 (3), 929-934. doi: 10.1161/ STROKEAHA.107.498725

Cung, T. T., Morel, O., Cayla, G., Rioufol, G., Garcia-Dorado, D., Angoulvant, D., et al. (2015). Cyclosporine before PCI in Patients with Acute Myocardial Infarction. N. Engl. J. Med. 373, 1021-1031. doi: 10.1056/NEJMoa1505489

Dagher, P. C., Herget-Rosenthal, S., Ruehm, S. G., Jo, S. K., Star, R. A., and Agarwal, R. (2003). Newly developed techniques to study and diagnose acute renal failure. J. Am. Soc. Nephrol. 14 (8), 2188-2198. doi: 10.1097/01. ASN.0000079790.91292.4A

Davidson, S. M., Ferdinandy, P., Andreadou, I., Bøtker, H. E., Heusch, G., Ibáñez, B., et al. (2019). Multitarget Strategies to Reduce Myocardial Ischemia/ Reperfusion Injury: JACC Review Topic of the Week. J. Am. Coll. Cardiol. 73, 89-99. doi: 10.1016/j.jacc.2018.09.086

De Vries, R. B. M., Wever, K. E., Avey, M. T., Stephens, M. L., Sena, E. S., and Leenaars, M. (2014). The usefulness of systematic reviews of animal experiments for the design of preclinical and clinical studies. ILAR J. 55 (3), 427-437. doi: 10.1093/ilar/ilu043

Deng, H. Y., and Lai, W. G. (2013). Protective Effect of Notoginsenoside R1 on Acute Myocardial Ischemia in Rats Model. Chin. J. Exp. Tradit. Med. Formulae (10). doi: 10.11653/syfj2013100265

Dong, X. H., Zhang, C. L., Wu, Z. G., Shen, L. X., Zhang, D. S., and Meng, X. Y. (2015). Protective effect of notoginsenoside R1 on cerebral ischemiareperfusion injury in rats. Chin. J. Gerontol. v.35 (07), 1940-1942. doi: 10.3969/j.issn.1005-9202.2015.07.093

Durukan, A., and Tatlisumak, T. (2007). Acute ischemic stroke: overview of major experimental rodent models, pathophysiology, and therapy of focal cerebral ischemia. Pharmacol. Biochem. Behav. 87 (1), 179-197. doi: 10.1016/j. pbb.2007.04.015

Egger, B., Van Giesen, L., Moraru, M., Sprecher, S. G. (2013). In vitro imaging of primary neural cell culture from drosophila. Nat. Protoc. 8 (5), 958-65 doi: $10.1038 /$ nprot.2013.052
Eltzschig, H. K., and Eckle, T. (2011). Ischemia and reperfusion-from mechanism to translation. Nat. Med. 17.11, 1391-1401. doi: 10.1038/nm.2507

Eltzschig, H. K., and Collard, C. D. (2004). Vascular ischaemia and reperfusion injury. Br. Med. Bull. 70 (1), 71-86. doi: 10.1093/bmb/ldh025

Fan, Q., Zhang, Y., Hou, X., Li, Z., Zhang, K., Shao, Q., et al. (2018). Improved oral bioavailability of notoginsenoside $\mathrm{r} 1$ with sodium glycocholate-mediated liposomes: preparation by supercritical fluid technology and evaluation in vitro and in vivo. Intl. J. Pharm. doi: 10.1016/j.ijpharm.2018.10.005

Feng, R., Liu, J., Wang, Z., Zhang, J., Cates, C., Rousselle, T., et al. (2017). The structure-activity relationship of ginsenosides on hypoxia-reoxygenation induced apoptosis of cardiomyocytes. Biochem. Biophys. Res. Commun., S0006291X1732034X. doi: 10.1016/j.bbrc.2017.10.056

Festing, M. F. W., and Altman, D. G. (2002). Guidelines for the design and statistical analysis of experiments using laboratory animals. Ilar J. 43 (4), 244. doi: 10.1093/ilar.43.4.244

Friedman, S. L., Sheppard, D., Duffield, J. S., and Violette, ,. S. (2013). Therapy for Fibrotic Diseases: Nearing the Starting Line. Sci. Transl. Med. 5 (167), 167sr1167sr1. doi: 10.1126/scitranslmed.3004700

Frobert, A., Valentin, J., Jean-Luc, M., Erwin, R., Cook, S., and Marie-Noëlle, G. (2015). Prognostic value of troponin i for infarct size to improve preclinical myocardial infarction small animal models. Front. Physiol. 6. doi: 10.3389/ fphys.2015.00353

Fu, Y., Yin, Z., and Yin, C. (2017). Biotransformation of ginsenoside Rb1 to ginsenoside Rg3 by endophytic bacterium Burkholderia sp. GE 17-7 isolated from Panax ginseng. J. Appl. Microbiol. doi: 10.1111/jam.13435

Furie, K. L., and Jayaraman, M. V. (2018). guidelines for the early management of patients with acute ischemic stroke. Stroke. doi: 10.1161/ STROKEAHA.118.020176

Galluzzi, L., Blomgren, K., and Kroemer, G. (2009). Mitochondrial membrane permeabilization in neuronal injury. Nat. Rev. Neurosci. 10 (7), 481-494. doi: $10.1038 / \mathrm{nrn} 2665$

Gashaw, I., Ellinghaus, P., Sommer, A., and Asadullah, K. (2012). What makes a good drug target? Drug Disc. Today 17 (supp-S), 0-0. doi: 10.1016/j.drudis.2011.12.008

Guo, Q., Li, P., Wang, Z., Cheng, Y., Wu, H., Yang, B., et al. (2014). Brain distribution pharmacokinetics and integrated pharmacokinetics of Panax notoginsenoside $\mathrm{r} 1$, ginsenosides $\mathrm{rg} 1, \mathrm{rb} 1$, re and $\mathrm{rd}$ in rats after intranasal administration of Panax notoginseng saponins assessed by uplc/ms/ms. J. Chromatogr. B. 969, 264-271. doi: 10.1016/j.jchromb.2014.08.034

Hacke, W., Donnan, G., Fieschi, C., Kaste, M., von Kummer, R., Broderick, J. P., et al. (2004). Association of outcome with early stroke treatment: pooled analysis of ATLANTIS, ECASS, and NINDS rt-PA stroke trials. Lancet 363, (9411). doi: 10.1016/S0140-6736(04)15692-4

Hadjidemetriou, M., Al-Ahmadi, Z., Mazza, M., Collins, R. F., and Kostarelos, K. (2015). In vivo biomolecule corona around blood-circulating, clinically-used and antibody-targeted lipid bilayer nanoscale vesicles. ACS Nano 9 (8), 81428156. doi: 10.1021/acsnano.5b03300

Hall, T. S., Hallén, J., Krucoff, M. W., Roe, M. T., Brennan, D. M., Agewall, S., et al. (2015). Cardiac troponin i for prediction of clinical outcomes and cardiac function through 3-month follow-up after primary percutaneous coronary intervention for st-segment elevation myocardial infarction. Am. Heart J. 169 (2), 257-265.e1. doi: 10.1016/j.ahj.2014.10.015

Han, J. Y., Li, Q., Ma, Z. Z., and Fan, J. Y. (2017). Effects and mechanisms of compound Chinese medicine and major ingredients on microcirculatory dysfunction and organ injury induced by ischemia/reperfusion. Pharmacol. Ther. 2017, S0163725817300852. doi: 10.1016/j.pharmthera.2017.03.005

Han, J. Y. (2014). The protective effect of Notoginsenoside R1 on the heart. Application Number: CN201310175976.8.

He, K., Yan, L., Pan, C. S., Liu, Y. Y., Cui, Y. C., Hu, B. H., et al. (2014). ROCKdependent ATP5D modulation contributes to the protection of notoginsenoside NR1 against ischemia-reperfusion-induced myocardial injury. Am. J. Physiol. Heart Circ. Physiol. 307, H1764-H1776. doi: 10.1152/ajpheart.00259.2014

Henderson, V. C., Kimmelman, J., Fergusson, D., Grimshaw, J. M., Hackam, D. G., and Ioannidis, J. P. (2013). Threats to validity in the design and conduct of preclinical efficacy studies: a systematic review of guidelines for in vivo animal experiments. PLoS Med. 10 (7), e1001489. doi: 10.1371/journal.pmed.1001489

Heusch, G., and Rassaf, T. (2016). Time to Give Up on Cardioprotection?: A Critical Appraisal of Clinical Studies on Ischemic Pre-, Post-, and Remote Conditioning. Circ. Res. 119 (5), 676-695. doi: 10.1161/CIRCRESAHA.116.308736 
Heusch, G. (2017). Critical Issues for the Translation of Cardioprotection. Circ. Res. 120 (9), 1477-1486. doi: 10.1161/CIRCRESAHA.117.310820

Heusch, G., and Gersh, B. J. (2016). The pathophysiology of acute myocardial infarction and strategies of protection beyond reperfusion: a continual challenge. Eur. Heart J. 38 (11), ehw224. doi: 10.1093/eurheartj/ehw224

Hietamies, T. M., Caroline, O., Zhong, P., Luyang, F., Christopher, M. C., Work, L. M., et al. (2018). Variability of functional outcome measures used in animal models of stroke and vascular cognitive impairment - a review of contemporary studies. J. Cereb. Blood Flow Metab., 0271678X1879985-. doi: 10.1177/0271678X18799858

Hou, Q. L., Wang, Y., Li, Y. B., Hu, X. L., and Wang, S. L. (2017). Protective effect of notoginsenoside R1 on neuron injury induced by OGD/R through ATF6/ Akt signaling pathway. Chin. J. Chin. Mater. Med. (6). doi: 10.19540/j.cnki. cjcmm.20170121.014

Jennings, R. B. (2013). Historical perspective on the pathology of myocardial ischemia/reperfusion injury. Circ. Res. 113 (4), 428-438. doi: 10.1161/ CIRCRESAHA.113.300987

Jones, P. A., May, G. R., Mcluckie, J. A., Iwashita, A., and Sharkey, J. (2004). Apoptosis is not an invariable component of in vitro models of cortical cerebral ischaemia. Cell Res. 14 (3), 241-250. doi: 10.1038/sj.cr.7290225

Joris, F., Manshian, B. B., Peynshaert, K., De Smedt, S. C., Braeckmans, K., and Soenen, S. J. (2013). Assessing nanoparticle toxicity in cell-based assays: influence of cell culture parameters and optimized models for bridging the in vitro-in vivo gap. Chem. Soc. Rev. 42 (21), 8339. doi: 10.1039/c3cs60145e

Judith, V. L., Brenda, B., Rovers, M. M., Merel, R. H., De, V. R. B. M., Marlies, L., et al. (2014). Systematic reviews of animal studies; missing link in translational research? PLoS ONE 9 (3), e89981-. doi: 10.1371/journal.pone.0089981

Kanelidis, A., Premer, C., Lopez, J. G., Balkan, W., and Hare, J. M. (2017). Route of delivery modulates the efficacy of mesenchymal stem cell therapy for myocardial infarction: a meta-analysis of preclinical studies and clinical trials. Circ. Res. 120 (7), 1139. doi: 10.1161/CIRCRESAHA.116.309819

Khandelwal, P., Yavagal, D. R., and Sacco, R. L. (2016). Acute ischemic stroke intervention. J. Am. Coll. Cardiol. 67 (22), 2631-2644. doi: 10.1016/j.jacc.2016.03.555

Kok, F., Shin, M., Ni, C. W., Gupta, A., Grosse, A., van Impel, A., et al. (2015). Reverse genetic screening reveals poor correlation between morpholinoinduced and mutant phenotypes in zebrafish. Dev. Cell 32 (1), 97-108. doi: 10.1016/j.devcel.2014.11.018

Lee, K. Y., Heo, J. H., Lee, S. I., and Yoon, ,. P. H. (2001). Rescue treatment with abciximab in acute ischemic stroke. Neurology 56 (11), 1585-1587. doi: 10.1212/WNL.56.11.1585

Leung, S. Y., Chan, K., Bensoussan, A., and Munroe, M. J. (2007). Application of atmospheric pressure chemical ionisation mass spectrometry in the identification and differentiation of Panax Species. Phytochem. Analysis 18 (2), 146-150. doi: 10.1002/pca.962

Li, C., Li, Q., Liu, Y. Y., Wang, M. X., Pan, C. S., Yan, L., et al. (2014). Protective effects of notoginsenoside $\mathrm{rl}$ on intestinal ischemia-reperfusion injury in rats. Am. J. Physiol. Gastrointest. Liver Physiol. 306 (2), G111. doi: 10.1152/ ajpgi.00123.2013

Li, Y., Yang, D., and Zhu, C. (2018). Impact of sodium n-[8-(2-hydroxybenzoyl) amino]- caprylate on intestinal permeability for notoginsenoside $\mathrm{r} 1$ and salvianolic acids in caco-2 cells transport and rat pharmacokinetics. Molecules 23 (11), 2990. doi: 10.3390/molecules 23112990

Liang, F. and Hua, J. X. (2005). Absorption profiles of sanchinoside R1 and ginsenoside Rg1 in the rat intestine. Eur. J. Drug Metab. Pharmacokinet. 30 (4), 261-268. doi: 10.1007/BF03190630

Liu, H., Yang, J., Du, F., Gao, X., Ma, X., Huang, Y., et al.. (2009). Absorption and disposition of ginsenosides after oral administration of Panax notoginseng extract to rats. Drug Metab. Dispos. 37 (12), 2290-2298. doi: 10.1124/ dmd.109.029819

Liu, W. J., Tang, H. T., Jia, Y. T., Ma, B., Fu, J. F., Wang, Y., et al. (2010). Notoginsenoside $\mathrm{rl}$ attenuates renal ischemia-reperfusion injury in rats. Shock 34 (3), 314-320. doi: 10.1097/SHK.0b013e3181ceede4

Liu, Z. j., Wang, H. Y., Hou, G. L., Cao, H. L., Zhao, Y., and Yang, B. F. (2019). Notoginsenoside R1 protects oxygen and glucose deprivation-induced injury by upregulation of miR-21 in cardiomyocytes. J. Cell. Biochem. 120, 9181-9192. doi: $10.1002 /$ jcb. 28194

Macarthur, J. W., Purcell, B. P., Shudo, Y., Cohen, J. E., Fairman, A., Trubelja, A., et al. (2013). Sustained release of engineered stromal cell-derived factor 1-a from injectable hydrogels effectively recruits endothelial progenitor cells and preserves ventricular function after myocardial infarction. Circulation 128 (11_suppl_1), S79-S86. doi: 10.1161/CIRCULATIONAHA.112.000343

Macleod, M. R., O'Collins, T., Howells, D. W., and Donnan, G. A. (2004). Pooling of animal experimental data reveals influence of study design and publication bias. Stroke 35 (5), 1203-1208. doi: 10.1161/01.STR.0000125719.25853.20

May, P. C., Dean, R. A., Lowe, S. L., Martenyi, F., Sheehan, S. M., Boggs, L. N., et al.. (2011). Robust central reduction of amyloid- $\beta$ in humans with an orally available, non-peptidic $\beta$-secretase inhibitor. J. Neurosci. 31, 16507-16516. doi: 10.1523/JNEUROSCI.3647-11.2011

Meng, X., Wang, M., Wang, X., Sun, G., Ye, J., Xu, H., et al. (2014). Suppression of nadph oxidase-and mitochondrion-derived superoxide by notoginsenoside r1 protects against cerebral ischemia-reperfusion injury through estrogen receptor-dependent activation of akt/nrf2 pathways. Free Radic. Res. 48 (7), 823-838. doi: 10.3109/10715762.2014.911853

Peng, M., Yi, Y. X., Zhang, T., Ding, Y., Le. J. (2018). Stereoisomers of Saponins in Panax notoginseng (Sanqi): A Review. Front. Pharmacol. 9, 188-. doi: 10.3389/ fphar.2018.00188

Murray Christopher, J. L. (2017). Global, regional, and national age-sex specific mortality for 264 causes of death, 1980-2016: a systematic analysis for the Global Burden of Disease Study 2016. Lancet 390, 1151-1210. doi: 10.1016/ S0140-6736(17)32152-9

Ng, T. B. (2006). Pharmacological activity of sanchi ginseng (Panax notoginseng). J. Pharm. Pharmacol. 58 (8), 1007-1019. doi: 10.1211/jpp.58.8.0001

Nishina, T., Nishimura, K., Yuasa, S., Miwa, S., Nomoto, T., Sakakibara, Y., et al. (2001). Initial effects of the left ventricular repair by plication may not last long in a rat ischemic cardiomyopathy model. Circulation 104 (Supplement 1), I-241-I-245. doi: 10.1161/hc37t1.094522

Oh, J., and Kim, J. S. (2016). Compound k derived from ginseng: neuroprotection and cognitive improvement. Food Funct. 7 (11). doi: 10.1039/C6FO01077F

Ohsawa, I., Ishikawa, M., Takahashi, K., Watanabe, M., Nishimaki, K., Yamagata, K., et al.. (2007). Hydrogen acts as a therapeutic antioxidant by selectively reducing cytotoxic oxygen radicals. Nat. Med. 13 (6), 688-694. doi: 10.1038/nm1577

Olesen, A. E., Andresen, T., Staahl, C., Drewes, A. M., et al. (2012). Human Experimental Pain Models for Assessing the Therapeutic Efficacy of Analgesic Drugs. Pharmacol. Rev. 64 (3), 722-779. doi: 10.1124/pr.111.005447

Overall, C. M., and Kleifeld, O. (2006). Tumour microenvironment-Opinion: Validating matrix metalloproteinases as drug targets and anti-targets for cancer therapy. Nat. Rev. Cancer 6 (3), 227-239. doi: 10.1038/nrc1821

Pelkonen, O., and Turpeinen, M. (2007). In vitro-in vivo extrapolation of hepatic clearance: biological tools, scaling factors, model assumptions and correct concentrations. Xenobiotica 37, 1066-1089. doi: 10.1080/00498250701620726

Perel, P., Roberts, I., Sena, E., Wheble, P., Briscoe, C., Sandercock, P., et al. (2006). Comparison of treatment effects between animal experiments and clinical trials: systematic review. BMJ 334. doi: 10.1136/bmj.39048.407928.BE

Powers, W. J., Rabinstein, A. A., Ackerson, T., Adevoe, O. M., Bambakidis, N. C., Becker, K., et al. (2018). guidelines for the early management of patients with acute ischemic stroke: a guideline for healthcare professionals from the american heart association/american stroke association. Stroke. 49 (3), e46e110. doi: 10.1161/STR.0000000000000158

Roberts, I., Kwan, I., Evans, P., and Haig, S. (2002). Does animal experimentation inform human healthcare? Observations from a systematic review of international animal experiments on fluid resuscitation. BMJ 324 (7335), 474476. doi: $10.1136 / \mathrm{bmj} .324 .7335 .474$

Ruan, J. Q., Leong, W. I., Yan, R., and Wang, Y. T. (2010). Characterization of metabolism and $\backslash \mathrm{r}$, in vitro $\backslash \mathrm{r}$, permeability study of notoginsenoside $\mathrm{r} 1$ from radix notoginseng. J. Agric. Food Chem. 58 (9), 5770-5776. doi: 10.1021/jf1005885

Sanne, J. O. L., Eding, J., Tycho, V. D. S., Vesterinen, H., Sena, E., Doevendans, P., et al. (2014). Similar effect of autologous and allogeneic cell therapy for ischemic heart disease: results from a meta-analysis of large animal studies. $J$. Am. Coll. Cardiol. 63 (12), A1762. doi: 10.1016/S0735-1097(14)61765-4

Sasaki, M., Kudo, K., Honjo, K., Hu, J. Q., Wang, H. B., and Shintaku, K. (2011). Prediction of infarct volume and neurologic outcome by using automated multiparametric perfusion-weighted magnetic resonance imaging in a primate model of permanent middle cerebral artery occlusion. J. Cereb. Blood Flow Metab. 31 (2), 448. doi: 10.1038/jcbfm.2010.106

Saulnier-Blache, J. S., Rory, ,. W., Kristaps, K., Delyth, G., Ioana, A., Kastenmüller, G., et al. (2018). Ldlr-/- and ApoE-/- mice better mimic the 
human metabolite signature of increased carotid intima media thickness compared to other animal models of cardiovascular disease. Atherosclerosis 276, 140-147. doi: 10.1016/j.atherosclerosis.2018.07.024

Seabrook, T. A., Burbridge, T. J., Crair, M. C., and Huberman, A. D. (2017). Architecture, function, and assembly of the mouse visual system. Annu. Rev. Neurosci. 40 (1), 499-538. doi: 10.1146/annurev-neuro-071714-033842

Sena, E. S., Currie, G. L., Mccann, S. K., Macleod, M. R., and Howells, D. W. (2014). Systematic reviews and meta-analysis of preclinical studies: why perform them and how to appraise them critically. J. Cereb. Blood Flow Metab. 34 (5), 737742. doi: $10.1038 / \mathrm{jcbfm} .2014 .28$

Shiva, S., Sack, M. N., Greer, J. J., Duranski, M., Ringwood, L. A., et al. (2007). Nitrite augments tolerance to ischemia/reperfusion injury via the modulation of mitochondrial electron transfer. J. Exp. Med. 204 (9), 2089-2102. doi: 10.1084/jem.20070198

Smith, C. (2003). Drug target validation: Hitting the target. Nature 422, 341, 343, 345 passim. doi: $10.1038 / 422341$ a

Su, P., Du, S., Li, H., Li, Z., Xin, W., Zhang, W., et al. (2015). Notoginsenoside R1 inhibits oxidized low-density lipoprotein induced inflammatory cytokines production in human endothelial EA.hy926 cells. Eur. J. Pharmacol. 770, 9-15. doi: 10.1016/j.ejphar.2015.11.040

Terkelsen, C. J., Sørensen, J.,. T., Maeng, M., Jensen, L. O., Tilsted, H. H., Trautner, S., et al. (2010). System delay and mortality among patients with stemi treated with primary percutaneous coronary intervention. JAMA 304 (7), 763. doi: 10.1001/jama.2010.1139

Thygesen, K., Alpert, J. S., Jaffe, A. S., Simoons, M. L., Chaitman, B. R., and White, H. D. (2012). Third universal definition of myocardial infarction. Circulation 126 (16), 2020-2035. doi: 10.1161/CIR.0b013e31826e1058

Tsujimoto, Y., Tsutsumi, Y., Kataoka, Y., Tsujimoto, H., Yamamoto, Y., Papola, D., et al. (2017). Association between statistical significance and time to publication among systematic reviews: a study protocol for a metaepidemiological investigation. BMJ Open 7 (10), e018856. doi: 10.1136/ bmjopen-2017-018856

Tsutsumi, Y. M., Tsutsumi, R., Mawatari, K., Nakaya, Y., Kinoshita, M., Tanaka, K., et al. (2011). Compound k, a metabolite of ginsenosides, induces cardiac protection mediated nitric oxide via akt/pi3k pathway. Life Sci. 88 (15-16), 0-729. doi: 10.1016/j.lfs.2011.02.011

Tu, L., Wang, Y., Chen, D., Xiang, P., and Wang, S. (2018). Protective effects of notoginsenoside 1 via regulation of the pi3k-akt-mtor/jnk pathway in neonatal cerebral hypoxic-ischemic brain injury. Neurochem. Res. 43 (9), 1210-1226. doi: 10.1007/s11064-018-2538-3

Van Hout, G. P. J., Jansen, O. L. S. J., Wever, K. E., Sena, E., Kouwenberg, L. H. J. A., Van Solinge, W. W., et al. (2015). Translational failure of anti-inflammatory compounds for myocardial infarction. Cardiovasc. Res. 109 (2), 240. doi: 10.1093/ $\mathrm{cvr} / \mathrm{cvv} 239$

Wan, Q., Pan, Y. Y., Jia, Y. H., and Liu, Z. Y. (2015). Notoginsenoside R1 Reduced Cardiomyocytes Injury Induced by Hydrogen Peroxide through JNK Signal Pathway. Lishizhen Med. Materia Med. Res. (9), 2061-2064. doi: 10.3969/j. issn. 1008-2015.09.005

Wang, L., and Ma, Q. (2018). Clinical benefits and pharmacology of Scutellarin: A comprehensive review. Pharmacol. Ther., S0163725818300809. doi: 10.1016/j. pharmthera.2018.05.006

Wang, Y., Tu, L., Li, Y., Chen, D., Liu, Z., Hu, X., et al. (2017). Notoginsenoside r1 alleviates oxygen-glucose deprivation/reoxygenation injury by suppressing endoplasmic reticulum calcium release via plc. Sci. Rep. 7 (1), 16226. doi: 10.1038/s41598-017-16373-7

Wang, Y., Tu, L., Li, Y., Di, C., and Wang, S. (2016). Notoginsenoside r1 protects against neonatal cerebral hypoxic-ischemic injury through estrogen receptordependent activation of endoplasmic reticulum stress pathways. J. Pharmacol. Exp. Ther. doi: 10.1124/jpet.115.230359

Wang, T., Guo, R., Zhou, G., Zhou, X., Kou, Z., Sui, F., et al. (2016). Traditional uses, botany, phytochemistry, pharmacology and toxicology of Panax notoginseng (Burk.) F. H. Chen: A review. J. Ethnopharmacol. 2016, S0378874116302665. doi: 10.1016/j.jep.2016.05.005

Wienkers, L. C., and Heath, T. G. (2005). Predicting in vivo drug interactions from in vitro drug discovery data. Nat. Rev. Drug Disc. 4 (10), 825-833. doi: 10.1038/ $\operatorname{nrd} 1851$
Worp, H. B. V. D., and Sandercock, P. A. G. (2012). Improving the process of translational research. BMJ 345, e7837-e7837. doi: 10.1136/bmj.e7837

Wozniak, A. W. (2004). Association of outcome with early stroke treatment: Pooled analysis of ATLANTIS, ECASS, and NINDS rt-PA stroke trials. Lancet 13 (5), 19. doi: 10.1016/j.accreview.2004.04.032

Xia, K. P., Cao, H. M., and Shao, C. Z. (2015). Protective effect of Notoginsenoside $\mathrm{r} 1$ in a rat model of myocardial ischemia reperfusion injury by regulation of vitamin d3 upregulated protein 1/nf-kb pathway. Die Pharm. Intl. J. Pharm. Sci. doi: $10.1691 / \mathrm{ph} .2015 .5694$

Yan, R. Q. (2016). Stepping closer to treating Alzheimer's disease patients with BACE1 inhibitor drugs. Transl. Neurodegener. 5 (1), 13. doi: 10.1186/ s40035-016-0061-5

Yang, X. C., Xiong, X.,. J., Wang, H.,. R., and Wang, J. (2014). Protective Effects of Panax Notoginseng Saponins on Cardiovascular Diseases: A Comprehensive Overview of Experimental Studies. Evid. Based Complementary Altern. Med. 2014, 1-13. doi: 10.1155/2014/204840

Yu, J. M., Ju, L., Wang, H. D., Wan, X. J., Yang, J. S., and Wang., Q. (2014). Effects of Notoginsenoside R1 on VEGF and bFGF Expression in Ischemic Myocardium of Rats. Prog. Mod. Biomed. 14 (30), 5845-5848. doi: 10.13241/j. cnki.pmb.2014.30.012

Yu, Y., Sun, G., Luo, Y., Wang, M., Chen, R., Zhang, J., et al.. (2016). Cardioprotective effects of notoginsenoside $\mathrm{rl}$ against ischemia/reperfusion injuries by regulating oxidative stress-and endoplasmic reticulum stress-related signaling pathways. Sci. Rep. 6, 21730. doi: 10.1038/srep21730

Zhao, Z. X., Hu, X. M., Wang, J., Cai, H. F. (2017). The effects of Notoginsenoside $\mathrm{R} 1$ on TNF- $\alpha$ mRNA after cerebral ischemia and reperfusion in rats. Zhejiang J. Trauma Surg. 22 (1). doi: 10.3969/j.issn.1009-7147.2017.01.005

Zhang, B., Zhang, J., Zhang, C., Zhang, X., Ye, J. X., Kuang, S. H., et al.. (2018). Notoginsenoside R1 Protects Against Diabetic Cardiomyopathy Through Activating Estrogen Receptora and Its Downstream Signaling. Front. Pharmacol. 9, 1227. doi: 10.3389/fphar.2018.01227

Zhang, B., Zhang, X. L., Zhang, C. Y., Shen, Q., Sun, G. B., and Sun, X. B. (2019). Notoginsenoside R1 Protects $\mathrm{db} / \mathrm{db}$ Mice against Diabetic Nephropathy via Upregulation of Nrf2-Mediated HO-1 Expression. Molecules 24. doi: 10.3390/ molecules2 4020247 undefined.

Zhang, S., Ju, Z., Guan, H., Yu, L., Wang, Z., and Zhao, Y. (2019). Dose-dependent exposure profile and metabolic characterization of notoginsenoside $\mathrm{R}$ in rat plasma by ultra-fast liquid chromatography-electrospray ionization-tandem mass spectrometry. Biomed. Chromatogr., e4670. doi: 10.1002/bmc.4670 undefined.

Zhou, F. H., Pan, Y. Y., Cui, X. B., and Jia, ,. Y.,. H. (2016). Notoginsenoside R1 Alleviates Cardiomyocytes Injury Induced By H2O2 Through ERK1/2 And p38 Signal Pathway. Chin. J. Herb. Pharmacology. (2), 17-20. doi: 10.13412/j. cnki.zyyl.2016.02.006

Zhou, F. H., Pan, Y. Y., Jia, Y.,. H., and Sun., X. G. (2017). Effect of Pim-2 in Cardiomyocytes Injury Induced by $\mathrm{H} 2 \mathrm{O} 2$ and Prevention of Notoginsenoside R1. Liaoning J. Tradit. Chin. Med. (03), 176-180. doi: 10.13192/j. issn.1000_1719.2017.03.056

Zhu, F., Shi, Z., Qin, C., Tao, L., Liu, X., Xu, F., et al.. (2012). Therapeutic target database update 2012: a resource for facilitating target-oriented drug discovery. Nucleic Acids Res. 40 (D1), D1128-D1136. doi: 10.1093/nar/gkr797

Zou, S., Zhang, M. X., Feng, L. M., Zhou, Y. F., Li, L., and Ban, L. L. (2017). Protective effects of notoginsenoside R1 on cerebral ischemia-reperfusion injury in rats. Exp. Ther. Med. 14, 6012-6016. doi: 10.3892/etm.2017.5268

Conflict of Interest: The authors declare that the research was conducted in the absence of any commercial or financial relationships that could be construed as a potential conflict of interest.

Copyright (c) 2019 Tong, Zhu, Zhuang, Deng, Wang, Zeng, Zheng and Wang. This is an open-access article distributed under the terms of the Creative Commons Attribution License (CC BY). The use, distribution or reproduction in other forums is permitted, provided the original author(s) and the copyright owner(s) are credited and that the original publication in this journal is cited, in accordance with accepted academic practice. No use, distribution or reproduction is permitted which does not comply with these terms. 